$U D K 536.7$

Rogankov V.B., Shvets M.V., Rogankov O.V.

Odessa National Academy of Food Technologies, Department of Physic and mat-hematic Sciences, Kanatnaya str., 112, 65039 Odessa, Ukraine

E-mail:vrogankov@yandex.ua

\title{
Mesoscopic metastable liquid in congruent vapor-liquid diagram of argon from about zero up to boyle`s temperature (review of FT-model).
}

Such paradigms of the coupled classical metastability and nonclassical criticality as the existence of a unified EOS (common for both gas and liquid phases) with its mean-field (mf), socalled Andrews-van der Waals' critical point (CP) should be questioned to recognize the realistic stratified structure of a mesoscopic liquid phase. It exists supposedly in the wide range of temperatures located between about zero $T \rightarrow 0, K$ and up to the singular first Boyle's point $T_{B}(\rho \rightarrow 0)$. Its opposite, also singular second Boyle's point $\rho_{B}(T \rightarrow 0, K)$ corresponds to the alternative origin for the crossover continuous bounds separating the specific structural strata of a mesoscopic liquid. The region of a heterogeneous l-phase spanning the whole temperature range can be termed the non-Gibbsian phase (due to its discrete cluster-like structure) without any appeals to the concept of a spinodal decomposition. The respective metastable liquid stratum is formed by three segments of supercritical $\left(T_{c}, T_{B}\right)$, subcritical $\left(T_{t}, T_{c}\right)$ and sublimation $\left(T \rightarrow 0, T_{t}\right)$ metastable states of a formally incompressible liquid constrained by the pair of fixed extensive parameters $(N, V)$. Its location on the CVL-diagram is restricted by the new introduced here ml-bound and by the known Zeno-line (ZL) bound. Thus, all above-mentioned strata belong to the region of soft fluid with the dominance of interparticle attraction. The remaining parts of CVL-diagram are spanned either by the real gas state-points and solid state-points (crystalline and/or amorphous) or by the region of hard fluid in the classification proposed by Ben-Amotz and Herschbach.

Key words: mesoscopic liquid, heterogeneous interphase, bounds of metastability.

I. Introduction. A variety of precise measurements in metastable vapor (v) and liquid (l) pha-ses fail to achieve the classical bounds predicted by the well-known spinodal locus. Its concept follows from the idealized notion of a homogeneous fluid $(f)$ Gibbsian phase supplemented by the Gibbs' phase rule and incorporated in the conventional Ehrenfest's classification scheme. For the first-order equilibrium phase transition, one assumes that three thermodynamic fields (temperature, pressure, chemical potential) of any two coexisting fluid $(f)$, gas $(g)$ or solid $(s)$ phases are strictly equal while the macroscopically uniform densities of mass, internal energy, entropy, etc. are discontinuous alongside the classical binodal. The classical WMGphenomenology developed by van der Waals, Maxwell and Gibbs for an equilibrium transition implies also the strict thermodynamic reversibility of condensation $(v \rightarrow l)$ and vaporization $(l \rightarrow v)$, solidification $(l \rightarrow s)$ and fusion $(s \rightarrow l)$ and of the other intermediate types of the transient processes.

These notes concern directly the problem of a realistic metastability in real substances with their finite-volume locally-inhomogeneous phases termed below as mesoscopic ones. Any transient processes within them occur during the finite time- 
intervals, which are unavoidably thermodynamically irreversible. Two conventional theoretical directions cannot be used to attack this complex problem. They are based either on the phenomenology of an equilibrium unified EOS $[1,2]$ or on the its direct simulation adopted in the two-phase region by the special methodology of a restricted ensemble $[3,4]$. We refer now an interested reader to the above-cited comprehensive investigations of classical i.e. homogeneous macroscopic metastability. The goal of the present work is to emphasize the crucial distinctions of classical concepts from the proposed recently methodology based on the concept of a congruent vapor-liquid (CVL) diagram [5, 6]. It will be used in the present work. This non-classical structural model of mesoscopic metastability realizes the previously formulated GFAprinciple of global fluid asymmetry [7-9].

GFA-principle [5,9] formulated in the framework of FT-model and its FT-EOS (FT-denotes the fluctuational thermodynamics [6,7]) rejects completely the classical WMG-concept [8] of a unified EOS not only at subcritical $\left(T^{-}<T_{c}\right)$ but also at supercritical $\left(T^{+}>T_{c}\right)$ temperatures. In particular, the direct consequence of the new fluctuational limit introduced for a metastable liquid $(\mathrm{ml})$ in the present work is the unusual location, shape and curvature of such metastable bound. It seems to be much more realistic in comparison with a classical spinodal due to the much more better correspondence with experiment. We believe that it well-established here continuous extension on the supercritical region and/or the extremely low temperatures will be interested for experimenters and theorists. For formers the revealed novel, more narrow density range of $m l$-states can be useful as a realistic alternative to the classical, experimentally unachievable spinodal. For latters, an argued absence of the traditional vdW-loop (see also simulated results of [4]) in CVL-diagram may be the serious stimulus for the further investigation of realistic non-classical metastability. In Sect. II we compare the traditional macroscopic VLE-diagram and the proposed mesoscopic CVL-diagram as the compatible «working tools» for the interpretation of metastable boundaries. Sects. III, IV provide the most essential details and results of GFA-principle applied, in particular, to argon. Sect. V contains the further discussion of the predictive abilities demonstrated by CVL-diagram in comparison with the most advanced theoretical and simulation methodologies developed recently for $f$-states. The most essential relevant results of the present work can be also found in the papers [68-70] published recently by V.Rogankov et al. in Physics of Airdispersive Systems journal.

II. Principle of global fluid asymmetry and concept of unified equation of state. Background of GFA-principle [7-9]. The most general form of FT-EOS contains $(T, f)$-dependent coefficients determined at any sub- and supercritical $\left(T^{-}, T^{+}\right)$temperatures but, separately, for two main $f$-phases of $g$ - and $l$-types $\left(Z_{f} \equiv P_{f} /\left(\rho_{f} k T_{f}\right)\right)$ : 


$$
Z_{F T}^{f}-1=\frac{b_{f}(T) \rho-c_{f}(T)}{1-b_{f}(T) \rho}-\frac{a_{f}(T) \rho}{k T} .
$$

Their crucial macroscopic distinctive feature is the different signs $(+/-)$ of an isothermal thermodynamic curvature. Hence, the presumable definition for their smoothly convergent isothermal $f$-segments corresponds to the bound of zero curvature $\left(\partial^{2} P / \partial \rho^{2}\right)_{T}=0$. This locus is well-known for a unified EOS as the curve of maxima for the isothermal compressibility $\chi_{T}(\rho)$ or the so-called fluctuation ridge. At the application of GFA-principle it should be determined alongside the locus of convergence for both $f$-phases. Third FT-coefficient $c_{f}(T)$ of an underlying thermodynamic imperfectness in a real finite-volume $(N, V)$-system takes into account the steady presence of the realistic thermodynamic fluctuations even in the extremely diluted but still real $g$-states. So the usual but physically questionable asymptotics of the deterministic ideal-gas (ig) model of a "complete vacuum" ( $Z^{i g} \rightarrow 1$ at $\rho \rightarrow 0, P \rightarrow 0$ ) have been replaced in Eq.(1) by the $f$-dependent trends for the fluctuational about ig-states at $\rho \rightarrow 0$ :

$$
Z_{F T}(\rho \rightarrow 0)=1-c_{f}(T) .
$$

Thus asymptotic first Boyle's $(B)$ temperature $T_{B}(\rho \rightarrow 0)$ in FT-EOS becomes singular in opposite to the conventional $B$-point. The latter is defined at the small but finite density. Its $\left(\rho, T_{B}\right)$-value for which $(\partial Z / \partial \rho)_{T_{B}}=0$ as $\rho \rightarrow 0$ [13] corresponds to the condition $B\left(T_{B}\right)=0$ for the second virial coefficient.

Two asymptotic values of the critical reduced slope $A_{c}$ (it is the equivalent of Riedel's similarity factor in the principle of corresponding states (PCS) [10-12]) are admissible by FT-EOS along the single the separate vapor-pressure $P_{g}(T) \approx P_{v}(T)$ branch complemented by the actual liquid-pressure $P_{l}(T)$-branch:

$$
A_{c}=\frac{T_{c}}{P_{c}}\left(\frac{d P_{g}}{d T}\right)_{c} \quad \text { (a) } \quad A_{c}^{0}=\frac{T_{c}}{P_{c}}\left(\frac{d P_{l}}{d T}\right)_{c}=4 \quad \text { (b). }
$$

The former corresponds presumably to the onset of condensation at any $T^{-} \leq T_{c}$. The latter is the vdW-assumption related to the onset of vaporization in a finite-volume $V$ determined for experimental or simulated $(N, V)$-system. The respective "bifurcation" of the critical point's (CP)-slopes from Eq.(3) is an essential factor for the construction of CVL-diagram in the supercritical region too. Its significance [6] for the correct development of any predictive methodology based on the usage of two asymptotic Boyle's $(B)$ parameters $T_{B}(\rho \rightarrow 0)$ and $\rho_{B}(T \rightarrow 0)$ should be now emphasized. Usually one admits [13-15] the strict linearity of so-called Zeno-line (i.e. $Z^{i g}=1$ ) in the $(T, \rho)$-plane to postulate then its predictive ability at the estimation of CP- 
parameters. Three main ingredients of such predictive (geometric and mean-field $(\mathrm{mf})$ by nature) procedure are:

1) the supposedly accurate knowledge [16] of both $T_{B}, \rho_{B}$-parameters follow-ing from the precise unified EOS with the long set of empirical coefficients of the type that $[17,18]$ developed for argon;

2) the crucial predictive role of the $m f$-rectilinear CXC-diameter;

3 ) the extrapolation, on the ad hoc basis, of the $B$-correlations derivable [13$15,19]$ from the original vdW-unified EOS with its $m f$-set of $C P$-parameters

$$
\begin{aligned}
\left\{T_{c}^{0}, P_{c}^{0}, \rho_{c}^{0} ; Z_{c}^{0}=3 / 8, A_{c}^{0}=4\right\}: & \\
Z_{v d W}-1 & =\frac{b \rho}{1-b \rho}-\frac{a b \rho}{b k T} \equiv \bar{\rho}\left(\frac{1}{1-\bar{\rho}}-\frac{1}{\bar{T}}\right) .
\end{aligned}
$$

on the transformed PCS-set of actual $Z_{c}$-dependent reduced CP-parameters:

$$
\omega=\rho / \rho_{c}=\bar{\rho} / Z_{c}, \quad \tau=T / T_{c}=9 \bar{T} Z_{c}, \quad \pi=P / P_{c}=9 \bar{P} / Z_{c} .
$$

Thus, one can recognize the finite $c_{f}\left(T_{B}\right)$ - coefficient from Eq.(2) as the factor of ZL-nonlinearity. Obviously, that the reliable empirical PCS-correlation [11] proposed long ago by Timmermans and implied by the first equality from the set of Eq.(5) is the necessary condition to connect the other vdW-estimates of reduced $B$ variables with the actual $\mathrm{CP}$-ones:

$$
\bar{\rho}=\frac{\rho}{\rho_{B}^{0}}=b \rho, \quad \bar{T}=\frac{T}{T_{B}^{0}}=\frac{b k T}{a}, \quad \bar{P}=\frac{P}{\rho_{B}^{0} k T_{B}^{0}}=\frac{P b^{2}}{a} .
$$

The noteworthy fact is here that the analogous $m f$-parametrization of the reduced LJ-variables are defined by only two supposedly known molecular parameters of the effective diameter $\sigma$ and the effective well-depth $\varepsilon[10,20]$ :

$$
\rho^{*}=\rho \sigma^{3}, \quad T^{*}=\frac{k T}{\varepsilon}, \quad P^{*}=\frac{P \sigma^{3}}{\varepsilon} .
$$

FT-model goes beyond the PCS-frameworks due to the exact CP-corre-lations [6-9] established for any $f$-dependent molecular effective parameters:

$$
\begin{gathered}
\varepsilon_{g}=\varepsilon_{l}=k T_{c}\left(1-Z_{c}\right)=k T_{c}-P_{c} / \rho_{c}, \\
\sigma_{g}^{3}=\frac{3\left(A_{c}-2\right)}{4 \pi \rho_{c}\left(A_{c}-1\right)} \quad(a) \quad \sigma_{l}^{3}=\frac{3\left(A_{c}^{0}-2\right)}{4 \pi \rho_{c}\left(A_{c}^{0}-1\right)}=\frac{1}{2 \pi \rho_{c}} \quad(b) .
\end{gathered}
$$

The important conclusion following from GFA-principle and from its basic Table 1 of the linear model-dependent CP-transformations is an unquestionable inherent correspondence between two main $m f$-models of a real $f$-state - vdW-and LJ-ones. We mean here that both ones may impart the underlying structure's features of any $f$ phase. For this aim one does not impose on its equilibrium with the other $f$-phase the supposedly necessary requirement of a continuous (i.e. unified) EOS. Contrariwise, if the whole two-phase $(N, V)$-system is still treated as a homogeneous Gibbsian $f$-phase with the continuously changeable density profile, WMG-phenomenology of a firstorder phase transition will always lead to the isothermal vdW-loop below the certain 
Table 1. Linear $\left(Z_{c}, A_{c}\right)$-dependent GFA-transformation of LJ-, PCS- and B-variables.

\begin{tabular}{|c|c|c|c|c|}
\hline \multicolumn{2}{|c|}{ f-phase *) } & LJ & PCS & B \\
\hline$g$ & $T^{*}$ & $\frac{k T}{\varepsilon}$ & $\frac{\tau}{1-Z_{c}}$ & $\frac{9 Z_{c}}{1-Z_{c}}$ \\
\hline$g$ & $\rho_{g}^{*}$ & $\rho_{g} \sigma_{g}^{3}$ & $\frac{3\left(A_{c}-2\right) \omega_{g}}{4 \pi\left(A_{c}-1\right)}$ & $\frac{3\left(A_{c}-2\right) \bar{\rho}_{g}}{4 \pi Z_{c}\left(A_{c}-1\right)}$ \\
\hline$g$ & $P_{g}^{*}$ & $\frac{P_{g} \sigma_{g}^{3}}{\varepsilon}$ & $\frac{3\left(A_{c}-2\right) Z_{c} \pi_{g}}{4 \pi\left(A_{c}-1\right)\left(1-Z_{c}\right)}$ & $\frac{27\left(A_{c}-2\right) \bar{P}_{g}}{4 \pi\left(A_{c}-1\right)\left(1-Z_{c}\right)}$ \\
\hline$g$ & $Z_{g}$ & $\frac{P_{g}^{*}}{\rho_{g}^{*} T_{g}^{*}}$ & $\frac{Z_{c} \pi_{g}}{\omega_{g} \tau}$ & $\frac{\bar{P}_{g}}{\bar{\rho}_{g} \bar{T}}$ \\
\hline$l$ & $T^{*}$ & $\frac{k T}{\varepsilon}$ & $\frac{\tau}{1-Z_{c}}$ & $\frac{9 Z_{c} \bar{T}}{1-Z_{c}}$ \\
\hline$l$ & $\rho_{l}^{*}$ & $\rho_{l} \sigma_{l}^{3}$ & $\frac{\omega_{l}}{2 \pi}$ & $\frac{\bar{\rho}_{l}}{2 \pi Z_{c}}$ \\
\hline$l$ & $P_{l}^{*}$ & $\frac{P_{l} \sigma_{l}^{3}}{\varepsilon}$ & $\frac{Z_{c} \pi_{l}}{2 \pi\left(1-Z_{c}\right)}$ & $\frac{9 \bar{P}_{l}}{2 \pi\left(1-Z_{c}\right)}$ \\
\hline$l$ & $Z_{l}$ & $\frac{P_{l}^{*}}{\rho_{l}^{*} T_{l}^{*}}$ & $\frac{Z_{c} \pi_{l}}{\omega_{l} \tau}$ & $\frac{\bar{P}_{l}}{\bar{\rho}_{l} \bar{T}}$ \\
\hline
\end{tabular}

$T_{c}^{0}$-boundary. This conclusion is independent on the accuracy to which the molecular-based imitation of a continuous $f$-state by the discrete $(N, V)$-system has been performed. One unavoidably needs (see, for example, polemic rising from the different simulated results of metastability in $[3,4]$ ) the explanation, from a physical viewpoint, for the appearance of the so-called equilibrium unstable $f$-states located within a spinodal. This fictitious, to our mind, notion cannot be attributed to a real fluid. However, it is widely discussable in the different PCS- and molecular-based theories [11, 12] as well as in the (essentially $m f$-ones by nature) simulations known under the term of a spinodal decomposition.

In the framework of GFA-principle [7-9], any real $(N, V)$-system should demonstrate the much less dramatic specific features in the entire two-phase range. This conclusion is in accordance with our preliminary unconstrained MD-simula-tions based on the introduced below short-range FT/LJ-potential (Sect. IV) with two main effective CP-dependent parameters $(\varepsilon, \sigma)$ from Eqs. $(8,9)$. Both rather narrow metastable ranges of density (in comparison with their spinodal's estimates) are separated by the relatively wide at low subcritical temperatures and about rectilinear but still slanting (i.e. non-horizontal in the $(P, \rho)$-plane) $v, l$-segments of $T^{-}$-isotherms. They corresponds naturally to the observable in a real fluid vapor-liquid $(v, l)$-mixture of 
small drops and bubbles. Such inhomogeneities steadily coexist, however, at the positive overall isothermal compressibility in the chosen control volume: $\bar{\chi}_{T}=(1 / \rho)(\partial \rho / \partial P)_{T}>0$. We suggest below the term mesoscopic FLUID for these realistic $f$-states located between the macroscopic GAS and LIQUID regions of gaussian fluctuations. Thus, the negative $\bar{\chi}_{T}$-value seems to be the artificial result following exclusively from a concept of unified EOS. In total, the strict WMG-conditions of a phase equilibrium between two Gibbsian $f$-phases cannot be accurately fulfilled in a real finite-volume $(N, V)$-system. Hence, the saturated pressure of real $l$-phase $P_{l}(T)$ should be always slightly higher than that in $g$-phase $P_{g}(T)$. We refer now the reader to the relevant problem of a thermodynamically small, mesoscopic $(N, V)$-system comprehensively studied by Hill [21] (see also Sect. IV).

Background of a unified EOS and classical metastability [1, 2]. The careful analysis of a spinodal's paradigm can be found in two consecu-tive reviews reported by Lienhard and co-authors [1,2]. To the best of our know-ledge, these relatively old works represent until now the state of the art, at least, in the problem of a unified EOS. We intend to demonstrate below that its discrepancy with the also unified scaling EOS of asymptotic criticality [22-24] can be removed not only by the conventional crossover Il/mf-phenomenology $[25,26]$ or by its global renormalization group (GRG)-expansion [27-29] on the entire $f$-range. Indeed, this fundamental problem is independently solvable in the framework of CVL-diagram (Sect. IV). The vdW-loop (inextricably linked to all above-named $m f$ - and non-classical scaling unified EOSs) never appears in FT-EOS (1) due to the mesoscopic [7-9] nature of many real $(N, V)$ systems (in which a volume $V$ is often less than a correlation one: $V \lessgtr \xi_{(c)}^{3}$ ). Nevertheless, the essential correspondence between the location of a new $\mathrm{ml}$-bound revealed below in the present work and that estimated by Fisher and Zinn [30] on the base of Ising-like (Il) criticality should be here emphasized. Both loci (i.e. the nonclassical spinodal [30] and the new $m l$-bound of CVL-diagram) lie appreciably closer either to the binodal for former or to the real $\mathrm{CXC}$ for latter than the original $m f$ spinodal of vdW-EOS.

The most appropriate for comparison and very accurate cubic LSB-EOS proposed by Lienhard, Shamsundar and Biney [1] has to be reduced, first of all, to the certain specific form. The aim is here the further compatibility with the GFAassumptions of Eq.(3). In terms of original PCS-denotations LSB-EOS is:

$$
\pi=\frac{\tau}{r+b}-\frac{a}{\tau^{\lambda}\left[(r+c)^{2}-d^{2}\right]},
$$

where the volume order parameter $\left(v-v_{c}\right) / v_{c}$ and the actual $Z_{c}$ are coupled by the spesific reduced density variable:

$$
r=Z_{c}\left(v-v_{c}\right) / v_{c}=Z_{c}(1-\omega) / \omega .
$$

Since both scaled so dimensional "densities" are coupled too: $P_{c} / k T_{c}=\rho_{c} Z_{c}$, 
one can add these CP-parameters to the adjustable Riedel's PCS-factor $\alpha_{R i}$ given in [1] by the following linear combination:

$$
\alpha_{R i}=\left.\frac{d \pi_{v}}{d \tau}\right|_{\tau=1}=\alpha(1+\lambda)-\lambda .
$$

All other LSB-coefficients are expressed in terms of the reference $\alpha$-parameter: $b=1 / \alpha, a=(1-b)^{3}, c=(1-b) / 2$ and $d^{2}=c^{2}(1-4 b)$.

The adjustable meaning of $\lambda$-parameter in Eq.(12) has been changed below by two accepted here FT-identities: $\alpha_{R i} \equiv A_{c}, \alpha \equiv A_{c}^{0}=4$ usable in Eq.(3). This replacement leads to the expressions of parameter $\lambda$ and LSB-EOS itself in the more appropriate for comparison forms:

$$
Z_{L S B}=\frac{4 Z_{c}}{4 Z_{c}(1-\omega)+\omega}-\frac{27 Z_{c} \omega}{64 \tau^{\lambda+1}\left[Z_{c}+\omega\left(Z_{c}^{0}-Z_{c}\right)\right]^{2}} .
$$

Thus the exponent $(\lambda+1)$ of a reduced temperature $\tau$ becomes $f$-dependent and has two distinct $f$-values (see Eq.(3)):

$$
\lambda_{g}+1=\left(A_{c}-1\right) /\left(A_{c}^{0}-1\right) \quad(a) \quad \lambda_{l}+1=1\left(\lambda_{l}=0\right) \quad(b) .
$$

To test the predictive capability of both equalities at the given CP-parame-ters and for the entire range of $(g, l)$-transition, the precise $\mathrm{CXC}$-data of argon tabulated in [18] have been substituted in Eq.(13). The very informative results of such substitution are represented in two variants by Figs. 1a, 1b. The failure of the wellconstructed, accurate and flexible but unified LSB-EOS [1] becomes evident from Fig. 1b obtained by substitution of the tabular $\omega_{l}(\tau)$-densities for Ar [18]. Even the sign of revealed curvature for the $P_{v}\left[\rho_{l}(T)\right]$-functional is wrong in this case because it resembles a spinodal instead of binodal.

It is straightforwardly to test also that the accepted and reliable [18] CP-values: $Z_{c}=0.2919$ and $A_{c}=5.943$ (see Fig. 1b) correspond only to the specific value $\lambda_{g}=0.6477$ by the reasonable extrapolation of the critical isochore $\omega=1$ :

$$
Z_{L S B}-1=4 Z_{c}-1-3 Z_{c} / \tau^{\lambda_{g}+1} \text {. }
$$

It gives the crossing with Zeno-line $\left(Z_{L S B}^{i g}=1\right)$ at the $B$-point $\tau_{B}=2.728$ (while the close value $\tau_{B}=2.627$ follows from Table 1$)$. On the other hand, the vdW-value of $\lambda_{l}=0$ from Eq.(14b) overestimates strongly $\tau_{B}$ by the same Eq.(15). Besides, the trend $\tau \rightarrow 0$ in Eq.(13) does not provide a possibility to obtain the respective $\omega_{B}$ estimate. It is in contradiction to the simple Timmermans' correlation: $\omega_{B}=3.426$ following from Table 1.

To revise the evident failure of a unified EOS at any efforts to describe reasonably and simultaneously both CXC-branches, one should be concerned, firstly, about the predictive description just of a single $l$-phase with its CP-position in accordance with the concept of CVL-diagram [5,6]. It is based exclusively on the measurable at 

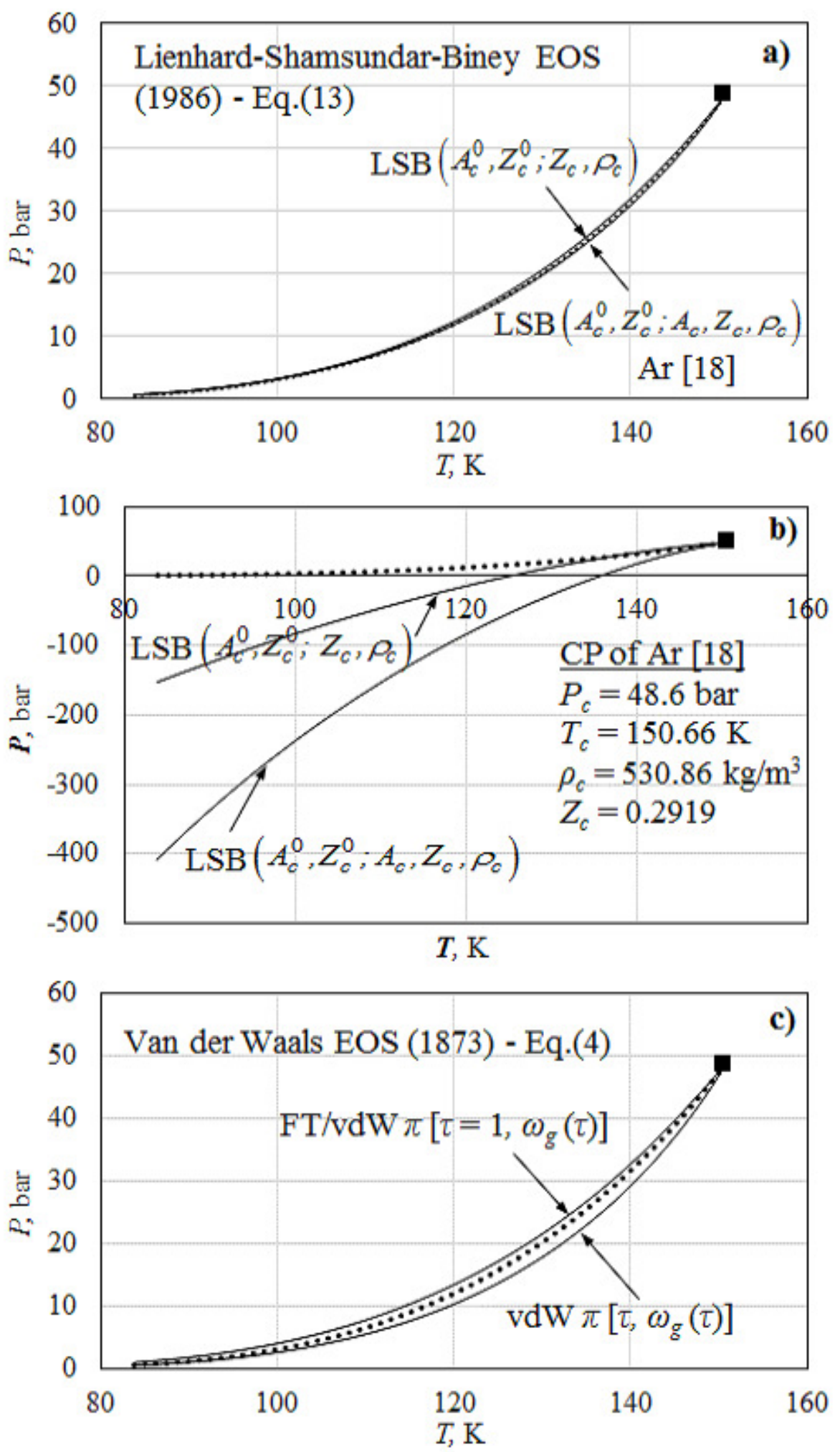

Fig. 1. Test of a vapor-pressure functional of density $P_{v}\left[\rho_{f}(T)\right]$ carried out either by substitution of saturated vapor $\rho_{g}(T)$ - tabular data for Ar [18] in the unified LSB-EOS (Fig.1a) or - of saturated liquid $\rho_{l}(T)$ - tabular data [18] (Fig.1b). Fig.1c compares the original vdW-EOS with its critical reduced isotherm $\tau=1$ used by FT/vdW-EOS to predict the saturated $\rho_{g}(T)$-branch [9]. 
atmospheric pressure $P_{0}$ input data of one-phase liquid $\rho\left(P_{0}, T\right)$. Then the more convincing and realistic than a spinodal's hypothesis procedure should be introduced to estimate the new locus of $m l$-limit. At last, the unique feature of the critical vdWisotherm $\tau=1$ illustrated by Fig. $1 \mathrm{c}$ has to be used to re-establish consistently with the predicted CP-position also the remaining $g$-branch.

III. Non-classical bound of metastable liquid ( $\mathrm{ml}$-limit) in macroscopic gaussian (g)-regime of CVL-diagram. The Gaussian ( $g$ )- and mesoscopic ( $m$ )regimes of fluctuations estimated by GFA-principle [5-9].

The ultimate predicted results of CVL-diagram for the macroscopic Gaussian [5] (i.e. thermodynamic) level of a finite-volume scale $V_{(g)}>V_{(c)}$ (its correlation estimate is denoted here by brackets) and for the underlying mesoscopic level $V_{(m)} \leq V_{(c)}$ of a discrete $(N, V)$-system should be in correspondence with the observable (i.e. measurable) thermodynamic properties of any $f$-states. The used subscripts in brackets $(g)$ and $(m)$ correspond to the above volume's scales termed by FT-model, in brief, Gaussian and mesoscopic regimes of a fluctuational behavior. Itself existence of the supposed correlation boundary $\left(N_{(c)}, V_{(c)}\right)$ between them for an equilibrium $(N, V)$-system implies that the given equilibrium $f$-point $\left.\rho_{f}=N / V \approx N_{(c)} / V_{(c)} ; T_{f}, P_{f}\right)$ belongs to the single-phase EOS-surface [31].

$\mathrm{CP}$ itself and its close asymptotic vicinity tend to the enormous numbers of the simultaneously correlated particles $N_{(c)}$ "immersed" in the unknown, a priori, correlation volume $V_{(c)}$. Due to this fundamental constraint of criticality, any thermophysical measurement or simulation near $\mathrm{CP}$ is naturally mesoscopic one (i.e.it belongs to $m$-regime). Hence, such less correlated $m$-volumes $V_{(m)}$ can be attributed either to stable or to metastable $f$-points of an EOS-surface $P(\rho, T)$ only if their thermodynamic bounds are previously estimated. The well-established results performed by Hill [21], Rowlinson and Widom [32-34], Penrose and Lebowitz [35], Scripov [36], Lienhard and co-authors [1, 2], Corti and Debenedetti [3] and by other investigators $[5,6,37-43]$ become relevant. Our aim below is to show that a common "Procrustean bed" of the unified EOS may be the serious restrictive feature of all above considerations especially in the extended CP-vicinity. The non-Gaussian behavior of non-Gibbsian $f$-phases is the most striking feature of $m$-regime. One needs the "tool" to take into account here the locally-heterophase fluctuations in a FLUID.

In particular, authors [3] have developed the appropriate technique of a restricted N,V,T-ensemble for the Monte Carlo algorithm in the framework of a standard coarse-grained analysis. Its specific feature is the choice, on the ad hoc basis, of the certain allowed limit for density fluctuations: $\delta N \geq \Delta N$ to imitate the about homogeneous (i.e. Gaussian by nature) $f$-state inside the metastable and even unstable regions. The upper bound on the severity of the $\delta$-fluctuation constraint has been determined by the statistical fluctuation equality $[22,23]$ : 


$$
\delta \equiv \frac{\left\langle(\Delta N)^{2}\right\rangle}{\bar{N}}=k T\left(\frac{\partial \rho}{\partial P}\right)_{T} \equiv \rho k T \chi_{T} .
$$

It was derived for an unconstrained single-phase system in the thermodynamic limit. The boundary value $\Delta N$ has been calculated by authors [3] for the saturated LJliquid branch $\rho_{l}(T)$ taken from the fundamental EOS constructed by Johnson et al [10]. More accurately, the separately simulated CXC-data for LJ-fluid, obtained previously by Lotfi et al [20], were incorporated in this rather complicated unified EOS as an auxiliary information. These preliminary CXC-data were approximated by the scaling-type empirical correlations, including those for $P_{v}^{*}\left(T^{*}\right)$-function.

We intend to demonstrate that the introduced by GFA-principle the bound rule of a unit fluctuation compressibility [5] in Eq.(16)

$$
\delta \equiv k T(\partial \rho / \partial P)_{T}=\left(\tau / Z_{c}\right)(\partial \omega / \partial \pi)_{\tau}=1
$$

provides the new very simple estimate of the realistic $m l$-limit. It corresponds to the inverse bulk modulus [44] which is independent on the absolute value of density. We extend this thermodynamic equivalent of the direct correlation function integral [5] to any $l$-states including supercritical ones. Their steady existence was corroborated by our FT/LJ-based MD-simulation and by its comparison [37,41] with the available experimental data on $l$-metastability $[1,2,36]$. The subcritical locus of $m l$-limit can be obtained without any appeals to the unachievable singular spinodal locus where: $\chi_{T} \rightarrow \infty$. In a finite-volume $(N, V)$-system the local isothermal compressibility $\chi_{T}$ and isobaric expansivity $\alpha_{P}$ should be always the finite quantities. An independent thermodynamic control of admissible metastable $l$-fluctua-tions has been provided by GFA-principle [5, 6, 9]. It was earlier applied not only to the widely usable for liquids reduced isothermal bulk modulus [44]:

$$
\left[\frac{\partial}{\partial \rho}\left(\frac{P_{f}}{k T}\right)\right]_{T} \equiv\left[\frac{\partial\left(Z_{f} \rho\right)}{\partial \rho}\right]_{T}=Z_{f}+\rho\left(\frac{\partial Z_{f}}{\partial \rho}\right)_{T} .
$$

but also to its isobaric "counterpart":

$$
\left[\frac{\partial}{\partial \rho}\left(\frac{P}{k T_{f}}\right)\right]_{P} \equiv\left[\frac{\partial\left(Z_{f} \rho\right)}{\partial \rho}\right]_{P}=Z_{f}+\rho\left(\frac{\partial Z_{f}}{\partial \rho}\right)_{P} .
$$

Let us note for comparison that the thermodynamic formalism adopted by the different variants of GRG-methodology [27-29, 45] is quite different:

$$
Z_{\text {rep }}=\frac{\rho}{k T}\left[\frac{\partial}{\partial \rho}\left(\frac{f_{r e p}}{\rho}\right)\right]_{T} \equiv \frac{\rho}{k T}\left[\frac{\partial}{\partial \rho}\left(\mu_{r e p}-\frac{P_{r e p}}{\rho}\right)\right]_{T} .
$$

It starts [27] from the singular purely repulsive hard-sphere $\left(h s^{-}\right)$model $Z_{\text {rep }}$ to switch then on the impact of a pair attraction $\phi_{\text {att }}(r)$ by the recurrent process. We have added the second identity in Eq.(20) to emphasize that the implied here Legendre transformation: $P(\mu, T)=\mu \rho-f(\rho, T)$ can be fulfilled, in principle, at any arbi- 
trary relationship between two unmeasurable (i.e. untestable by experiment) quantities of the Helmholtz's free energy $f=F / V$ per unit volume and the Gibbs' chemical potential $\mu=G / N$ per particle. This "element of uncertainty" makes the overall set of GRG-iterations to be implicitly dependent on the choice of input physical model. In this context, the use of any locally-singular imitation of a continuous fluid behavior by the hard- or soft-spheres (ss-) the rectangular (square) or triangular wells ( $s w$ - and $t w$-), the infinitely weak and infinitely long-ranged (Kac's type potential) or finitely strong and short-ranged (Yukawa's type potential) etc. is not a completely satisfactory choice from the physical viewpoint. Such oversimplification leads not only to the artificial "symmetrization" of a GRG-predicted CXC for real fluids [28, 29]. Another undesirable consequence is the serious uncertainty in two main potential parameters $(\varepsilon, \sigma)$ arisen due to the model-dependent appearance of a third parameter (see Sect. IV).

Instead of an approximated free-energy $(f(\rho, T) / \rho)$-functional of Eq.(20) used to derive the GRG-methodology [27], both introduced by Eqs. $(18,19)$ functionals can be specified along the actual coexisting CXC-branches of a fluid: $Z_{g}\left[\rho_{g}(T)\right]$ and $Z_{l}\left[\rho_{l}(P)\right]$. They tend at the parallel trends $\rho_{f} \rightarrow \rho_{c}$ to the common CP-value $Z_{c}$. Let us note that this main PCS-factor $[11,12]$ was used in GRG-procedure only as the input known parameter, at the determination of CP-location. In contrast to all intents and purposes of its authors, the adopted GRG-criterion for finding CP-isotherm $T=T_{c}$ [27] at the given actual $Z_{c}$ as the curve with an inflection point (it determines CP-density $\rho_{c}$ ) and with the horizontal tangent $C P$-isobar $P_{c}$ is an essentially $m f$ one by nature:

$$
(\partial P / \partial \rho)_{T_{c}^{0}, \rho_{c}^{0}}=0 \quad(a) \quad\left(\partial^{2} P / \partial \rho^{2}\right)_{T_{c}^{0}, \rho_{c}^{0}}=0 \quad(b) .
$$

Such classical definition of the so-called Andrews-van der Waals' CP [32] is legitimate only if the concept of a unified EOS has been implicitly adopted.

GFA-principle introduces the alternative criterion to determine an unknown CPlocation and, mainly, its $\rho_{c}$-value [6] by the system of two asymptotically divergent CP-equalities:

$$
\begin{aligned}
\chi_{T_{c}} & =\frac{Z_{c}}{P_{c}} \cdot \frac{1}{\left[Z_{g}+\left(\partial Z_{g} / \partial \rho\right)_{T_{c}} \rho\right]_{\rightarrow 0}}, \\
\alpha_{P_{c}} & =\frac{Z_{c}}{T_{c}} \cdot \frac{1}{\left[Z_{l}+\left(\partial Z_{l} / \partial \rho\right)_{P_{c}} \rho\right]_{\rightarrow 0}} .
\end{aligned}
$$

Hence, the $m f$-CP determined by Eq.(21) never appears in CVL-diagram.

CVL-diagram and locus of ml-limit predicted in g-regime. In the practical application of the Gibbs' differential thermodynamic forms (fir instance, to produce the non-equilibrium trajectories by integration) one needs an adequate EOS in the whole region of $f$-states. It is of great importance to predict with a reasonable accuracy the 
experimentally unknown thermophysical properties far away from the wellrecognized ranges: $\left(T_{c}, T_{t}\right)$ and $\left(P_{c}, P_{t}\right)$ of VLE-diagram. The proposed below methodology of CVL-diagram maps out concrete measures to realize such a promising plan. Its essential ingredient and a first step is the construction of a realistic CVLsketch in $g$-regime for its further specification in the whole $f$-range of interest.

An example of CVL-diagram shown in Fig. 2 for Ar combines the main experimental projections of its VLE-diagram [18] represented by symbols with the respective projections of the following four loci: 1) classical spinodal (sp): $\left.(\partial \bar{P} / \partial \bar{\rho})_{\bar{T}}=0 ; 2\right)$ classical quasi-spinodal [46] $\left.(q s p):\left(\partial^{2} \bar{P} / \partial \bar{\rho}^{2}\right)_{\bar{T}}=0 ; 3\right)$ new metastable liquid $(m l)$-limit: $\left.(\partial \bar{P} / \partial \bar{\rho})_{\bar{T}} / \bar{T}=1 ; 4\right)$ classical Zeno-line $(\mathrm{ZL}): \bar{P} /(\bar{\rho} \bar{T})=1$. The use of $B$-variables (Table 1 ) and the choice of basic vdW-EOS (4) for $l$-phase are restricted in $g$-regime by the equality $A_{c}^{0}=4$ from Eq.(3b) while $Z_{c}$-value is actual ( $Z_{c}^{A r}=0.2919$ [18]). The latter is necessary to convert by Eqs.(5) the respective equations of above loci into PCS-variables of Fig. 2:

$$
\begin{aligned}
& \bar{T}_{s p}=2 \bar{\rho}(1-\bar{\rho})^{2} \\
& \bar{T}_{q s p}=(1-\bar{\rho})^{3} \\
& \bar{T}_{m l}=\frac{2(1-\bar{\rho})^{2}}{2-\bar{\rho}} \\
& \bar{T}_{Z L}=1-\bar{\rho}
\end{aligned}
$$$$
\bar{P}_{s p}=\bar{\rho}^{2}-2 \bar{\rho}^{3}
$$$$
\text { (b) , }
$$$$
\bar{P}_{q s p}=\bar{\rho}(1-3 \bar{\rho})+\bar{\rho}^{3}
$$

$$
\begin{gathered}
\bar{P}_{m l}=\frac{2 \bar{\rho}(1-2 \bar{\rho})+\bar{\rho}^{3}}{2-\bar{\rho}} \\
\bar{P}_{Z L}=\bar{\rho}(1-\bar{\rho}) \quad(b) .
\end{gathered}
$$

We suppose that the collected here bounds provide a realistic information for the further detailed study of any simple or complicated substances in the whole range of its aggregate $g-, l-, f-, s$-states. Let us remind, once more, that the final knowledge of CVL-diagram in CP-vicinity should include the peculiarities of $m$-regime (Sect. IV). Besides, the reliable information about GAS and FLUID regions shown in Fig. 2 cannot be obtained without the asymptotic $A_{c}$-slope for $P_{g}(T) \approx P_{v}(T)$-branch and without the additional $P_{l}(T)$-branch originating from the actual CP (Sect.II). Therefore, we have depicted the standard VLE-diagram by diamond-symbols and the actual CP - by black-square to outline, in particular, a predictive strategy if these CXCdata are unknown. In this case, the role of CVL-diagram, a priori predicted by Eqs.(27-30), becomes especially valuable.

We consider three congruous domed qsp-, $m l-, Z L$-loci in the $(\pi, \tau)$ - and $(\pi, \omega$ )-projections as the basic ones for the construction of CVL-diagram. The classical $s p$ locus is, at best, the subsidiary one. Indeed the formal role of latter is re-stricted by its crossing with $q s p$-locus to predict the Andrews-van der Waals $m f$-CP in accordance with its classical definition by Eq.(21). Itself thermodynamic existence of such point of intersection became recently the object of an aggressive polemic [47, 48] between Woodcock and some adepts of scaling phenomenology. The first author revealed (erroneously from the viewpoint of GFA-principle but rather plausibly in the context of empirical, very precise unified EOS of the type $[17,18]$ ) an wide two-di- 

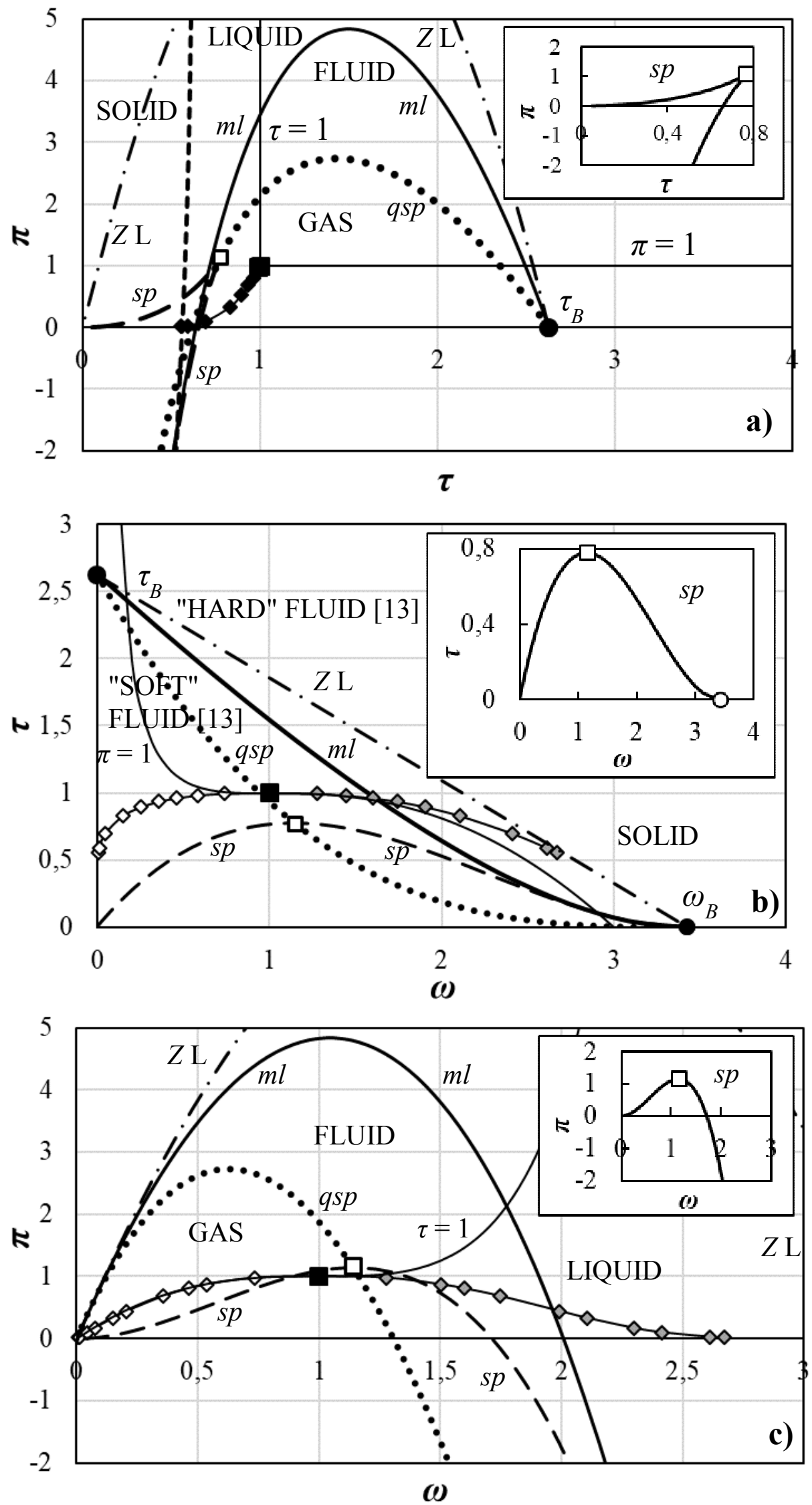

Fig. 2. CVL-diagram for Ar in $g$-regime formed by its basic loci ( $q s p, m l, Z L-$ see comments in text) and comparison with the classical spinodal ( $s p-$ see insets and dashed lines) imposed on the experimental VLE-diagram. 
mensional region of supercritical $q s p$-states in the $(P, \rho)$-plane termed the mesophase by him [48]. Its "bottom", in accordance with such phenomenology, corresponds to the percolation density range of a critical coexistence line formed by the special set of $s p$-states instead of a single $m f$-CP. This concept has been seriously criticized [48] but, unfortunately, in the same framework of a unified EOS hypothesis applied to the non-classical criticality. Just the classic WMG-hypothesis is the main obstacle, from our viewpoint, to reconcile the above controversial arguments in which authors confuse the quite different notions of actual $\mathrm{CP}$ and $m f$-CP. In accordance with Fig. 2a) the first point shown by black square is really the point of intersection for two critical loci $\tau=1$ and $\pi=1$ (Woodcock rejects such a possibility) but it becomes the point in which both curves are tangent one to another in two other projections $2 b), 2 c)$. At the same time, the Gibbs' phase rule is really in contradiction (Woodcock is right in this claim) to the fictitious $m f$-CP (white square). Moreover, CVL-diagram provides the evident alternative explanation for the percolation peculiarities called the supercritical mesophase in $[47,48]$. We have used the more habitual term FLUID for the region of supercritical $f$-states located between $q s p$ - and $m l$-loci. In other words, this region with the actually negligible but still finite positive curvature $\left(\partial^{2} P / \partial \rho^{2}\right)_{T^{+}} \geq 0$ and the positive compressibility $(\partial P / \partial \rho)_{T^{+}}>0$ separates in CVL-diagram GAS from metastable (supercritical here) LIQUID. The evident fluctuation flattening of the experimental CXC-top is especially expressive in the $(\tau, \omega)$-plane.

There are three fundamental $B$-points of a substance well-established by the asymptotic trends of all basic loci in CVL-diagram. The first is $B$-temperature: $\tau_{B}=2.625(\omega \rightarrow 0)$ which gives the realistic estimate: $T_{B}=395.8 \mathrm{~K}$ for $\operatorname{Ar}[11,16]$. The second is $B$-density $\omega_{B}=3.426(\tau \rightarrow 0)$, which corresponds to the value $\rho_{B}=1818.6 \mathrm{~kg} / \mathrm{m}^{3}$ for Ar. The mechanical $s p$-locus tends asymptotically to the $B$ point $\omega_{B}(\tau \rightarrow 0)$ too. At last, the common third asymptotic point of the ne gative $B$ pressure: $\pi_{B}=-30.832\left(\tau \rightarrow 0 ; \omega \rightarrow \omega_{B}\right)$ corresponds to the quite realistic [36] estimate: $P_{B}=-149.8 \mathrm{MPa}$ for Ar. The dashed bold line of $l, s$-transition shown only in Fig. 1a) has been re-constructed in the present work by the semiempirical Simon's correlation [11] on the base of this $P_{B}$-value.

Fig. 3 in which the predicted $Z_{c}$-dependent (see Eq.(17)) $m l$-loci are shown for the set of very different substances emphasizes the GFA-universality going beyond the PCS-frameworks $[11,12]$. Its another aim is to demonstrate the indicative value just of l-branch $\rho_{l}\left[P_{l}(T)\right]$ represented for comparison by the experimental CXCdata used earlier for its discussion in [9]. In particular, one may see from two variants of the Guggenheim's type [22] projections on the $(\tau, \omega)$ - and $(\pi, \omega)$-planes, that namely the set of $l$-branches is evidently stratified for such groups as the PCS-similar substances $\mathrm{Ar}$ and $\mathrm{C}_{2} \mathrm{H}_{6}$, from one hand, and the molecular $\mathrm{H}_{2} \mathrm{O}$, from the other hand. The fundamental meaning of all $\mathrm{ml}$-loci becomes apparent since their about common crossover point (shown in Fig. 3a) by the white circle) divides accurately l-branch 

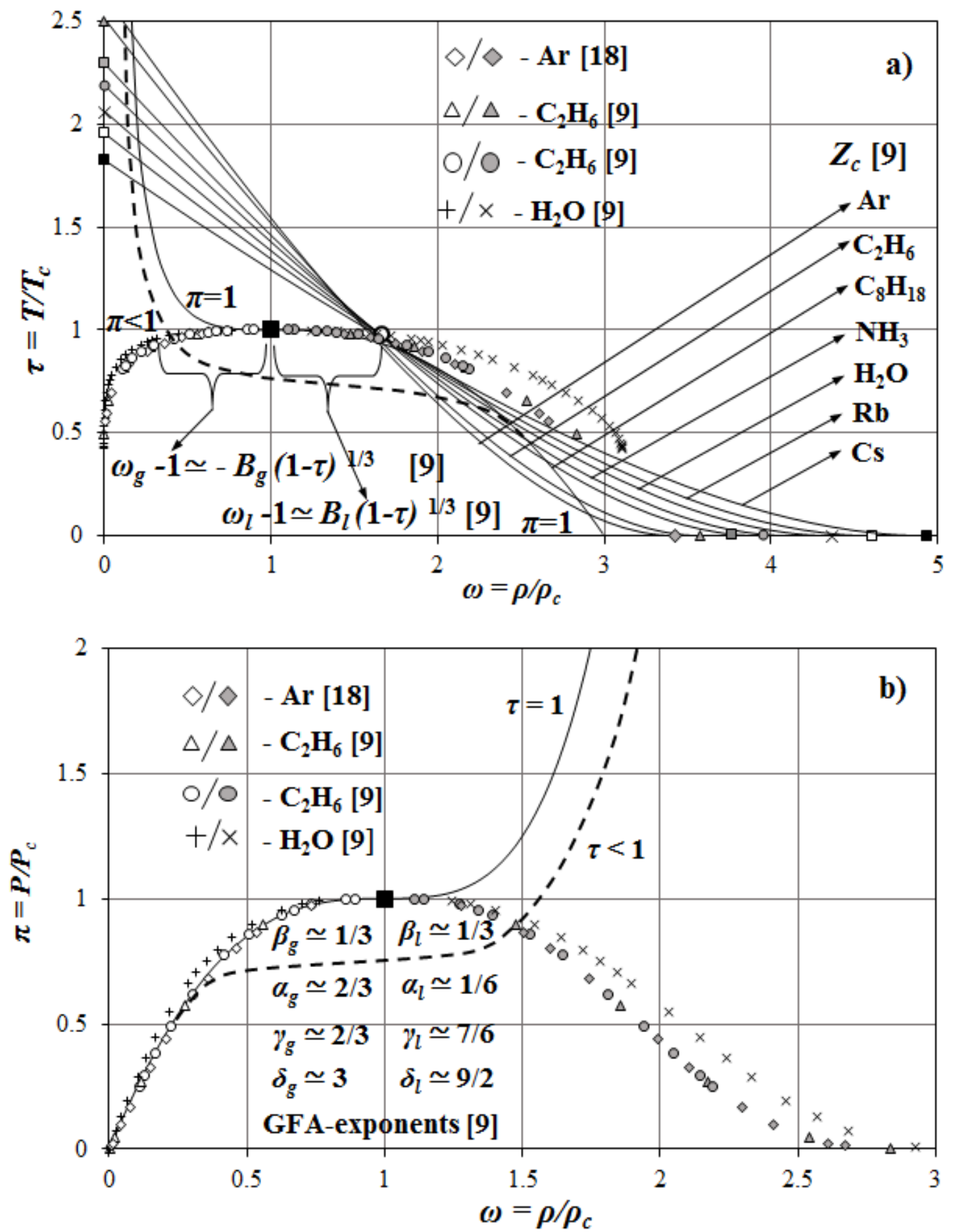

Fig. 3. The predicted by CVL-diagram $m l$-boundaries of a supercritical $(\tau<1)$, subcritical $\left(1 \geq \tau \geq \tau_{t}\right)$ and low-temperature $\left(0<\tau<\tau_{t}\right)$ metastable liquid are compared with the experimental CXC-data for $\mathrm{Ar}, \mathrm{C}_{2} \mathrm{H}_{6}, \mathrm{H}_{2} \mathrm{O}$.

3a) The reduced critical vdW-isobar $\left(\pi=P / P_{c}=1\right)$ is the envelop (caustics) of all subcritical isobars $(\pi<1)$ (one of them is schematically represented in Fig. 3a by the dashed line). 3b) The reduced critical vdW-isotherm $\left(\tau=T / T_{c}=1\right)$ is the envelop (caustics) of all subcritical isotherms $(\tau<1)$ (one of them is schematically represented in Fig. $3 \mathrm{~b}$ by the dashed line). Any unstable f-states cannot be revealed in a finite-volume closed $(N, V)$-system by the adequate experiment or simulation (GFAconclusion). 
onto the region of universal criticality and the region of its substance-dependent behavior. Thus, it is naturally to use this feature below to specify any simple or complex molecular force-field. The collected in Fig.3b) GFA-estimates of critical exponents for CXC correspond, in total, to the known Griffiths-Wheeler's classification of near-critical isolines $[49,50]$.

\section{$I V$. Peculiarities of pseudo-homogeneous criticality in m-regime and crosso-} ver problem. Existence of non-Gibbsian locally-heterogeneous FLUID-phase in supercritical m-regime and VAPOR-phase in subcritical $m$ - and g-regimes.

There were many long-standing and recent interesting efforts [13-15, 24-29, 4659] to specify the different structural types of SCF (supercritical fluid)-behavior in the extended so-called compressible fluid region. Even this term seems to be rather elusive. As a rule, the main goal of such specification is a search for the certain line of the thermodynamic supercritical continuation in the $(P, T)$-plane. It exists supposedly for the unique at subcritical $T^{-}$-temperatures vapor-pressure curve $P_{v}(T)$. There are several well-known "candidates" for this aim related by different authors to the famous names of Semenchenko [46] (quasispinodal ( $q s p$ ) or the line of inflection points), Bernal [51] (hypercritical line of the maximum for isobaric heat capacity $\left.C_{P}=(\partial h / \partial T)_{P}\right)$, Widom [52-54] (generalized near-critical locus of the maxima for $C_{P}$, isothermal compressibility $\chi_{T}=(1 / \rho)(\partial \rho / \partial P)_{T}$ and isobaric expansivity $\left.\alpha_{P}=-(1 / \rho)(\partial \rho / \partial T)_{P}\right)$ ), Frenkel [55] (dynamic boundary between the rigid liquid and nonrigid liquid). The latter nomenclature resembles formally one introduced by Ben-Amotz and Herschbach [13] to separate "hard" fluid from "soft" fluid by Zenoline in $(\tau, \omega)$-plane (see Fig. 2b). However, the Frenkel line bears even the more impressive resemblance with the $m l$-locus introduced in the present work. Indeed, both are not related to the actual CP-position. Nevertheless, their slopes in the $(\tau, \omega)$-plane are opposite one to another.

The $m l$-locus as well as two other basic curves (qsp- and ZL-loci) of CVLdiagram realize the GFA-concept of a longitudinal crossover connecting two asymptotic Boyle's points (Sec. II) in all EOS-projections. The inhomogeneous cluster-like structure of a region constrained by $q s p$ - and $m l$-loci in Fig. 2 manifests itself only in $m$-regime at $\left(T^{+} \geq T_{c}\right)$-conditions while in $g$-regime it can be formally considered as the Gibbsian phase. However, the same region demonstrates at subcritical $\left(T^{-}<T_{c}\right)$ conditions the steady percolation structure of a vapor-liquid non-Gibbsian phase composed by voids and clusters in $g$-regime too. In contrast, majority of the different crossover variants discussed in above-cited works can be termed a transversal crossover in which the density becomes the main transformation parameter instead of temperature.

As a rule, the foundation adopted in this case for the crossover identification is the model-dependent and, hence, non-universal. More accurately, to specify, for example, the location of dynamic bound between rigid and nonrigid liquid one adopts 
[55] the balance condition for the kinetic energy per particle $E_{k i n} / N$ and its mean potential energy $E_{p o t} / N$. The latter is determinable only by the nearest $N_{c}$ particles-neighbors located withi the certain cutoff radius: $r \leq r_{c}$ :

$$
E_{k i n} / N=3 k T / 2 \approx\left(N_{c}-1\right) \sum_{i, j} \phi_{i, j}\left(r \leq r_{c}\right) / 2 .
$$

An attempt to confirm the universality of such finding was performed by two antipodal models of $f$-states: 1$)$ the realistic LJ-fluid with the cutoff radius $r_{c}=2.5$; 2) the singular and unrealistic (at least, in a range of "soft" fluid (Fig. 2b) with the dominance of attraction) purely repulsive model of $s s$-fluid. The serious restrictive feature of $s s$-model (studied comprehensively long ago by Hoover and co-authors [60]) is an absence of VLE-transition. Supposedly, the similar highly-modelistic absence of VLE-transition should be also demonstrated by the very "narrow and deep" singular attraction of the type that introduced in a combination with $h s$-model by $\mathrm{Yu}$ kawa at the description of atomic nuclei [61].

From the viewpoint of CVL-diagram, any molecular-based crossover vari-ants have to be controllable by the realistic thermodynamic EOS-model $P(\rho, T)$ without an appeal to the model-dependent, unmeasurable equilibrium chemical potential $\mu(P, T)$ and free energy $f(\rho, T)$ (Sect. III). In other words, one should prefer to supplement the $P(\rho, T)$-description with analysis of a crossover problem in terms of the measurable caloric EOS $e(T, P)$ for thermal (internal) energy. This function is expressed in the FT-model, by its non-natural thermodynamic variables because in accordance with the Gibbs' formalism: $e=e\left[s=-(\partial \mu / \partial T)_{P}, v=(\partial \mu / \partial P)_{T}\right]$ :

$$
C_{P} \equiv\left(\frac{\partial h}{\partial T}\right)_{P}=\left(\frac{\partial e}{\partial T}\right)_{P}+P\left(\frac{\partial v}{\partial T}\right)_{P} \equiv\left(\frac{\partial e}{\partial T}\right)_{P}+P v \alpha_{P} .
$$

Such substitution of an actual measurable enthalpy $h(T, P)$ instead of its entropydependent thermodynamic "counterpart": $h=h(s, P)=e(s, v)+P v$ seems to be highly desirable, especially, in $m$-regime where the serious problem arises. This is an inherent dependence of all thermodynamic fields $\mu ; P, T$ on the arbitrarily chosen extensive parameter ( $N$ or $V$ ) in such traditional statistical ensemble [23] as the isobaric $(N ; P, T)$ and/or grand-canonical $(V ; \mu, T)$ ones. The study of "incompressible $(N, V)$ systems" alongside the fixed isochore: $\rho=N / V$ becomes, in this context, preferable dust in $m$-regime.

The FT-model developed earlier [7-9] and used in the present work excludes the itself traditional WMG-notion of an isotherm-isobar determined as a line of a phase transition with the continuously variable densities. The latter leads not only to the formal divergences of compressibility $\chi_{T}$ and expansivity $\alpha_{P}$ everywhere within the classical $m f$-binodal. Indeed, the choice of the standard grand-canonical $(V ; \mu, T)$ or isobaric $(N ; P, T)$-ensembles with two fixed coordinate-fields cannot define comprehensively [32] the state of two-phase or, generally, heterophase assembly. Both pairs 
of their conjugated momenta-densities: $\rho, \sigma=\rho s$ or specific (per mole or per particle) quantities: $v, s$ may vary locally within the given limits without changing of the fixed fields: $\mu, T$ or $P, T$ respectively. In other words, the transformation of the measureable $P, v, T$ EOS-surface into that determined exclusively by the fields: $P, \mu, T$ Gibbsian-surface (see, for example [48, 49]) might, in principle, "wash off" the realistic heterophase structures of real fluids and their mixtures.

Thermodynamics assumes a possibility of the Gibbsian (i.e. macroscopic) isothermal differentiation in $g$-regime applied to the thermodynamic fields of pressure $P$ and chemical potential $\mu$. The latter is determined for a pure substance as the specific (per particle) Gibbs' uniform function: $G=N \hat{\mu}(T, P)$. The methodology of a unified EOS postulates that its non-natural variable of the mean (i.e. uniform) number density $\rho=N / V=1 / v$ can be used as independent one. The aim is either to realize the known Maxwell's rule at $T^{-}<T_{c}$ or to define the "extensive state function of the mesophase rigidity" $R_{m}$, for example, proposed in [48] at $T^{+} \geq T_{c}$ :

$$
(d P / d \rho)_{T \geq T_{c}}=\rho(d \mu / d \rho)_{T \geq T_{c}}=R_{m}\left(T^{+}\right) .
$$

This conventional local $\rho$-parametrization of the Gibbs-Duhem's differential form is based on the implied assumption of a smooth EOS-surface $\rho(P, T)$. It becomes incorrect one (due to the inconsistent choice of independent variable) if the finite-volume small $(N, V)$-systems are composed by the small number of constituent particles within $m$-regime. In this case, one has to distinct [21] the uniform abovementioned chemical potential $\hat{\mu}=G / N$ from its local differential form $\mu=(\partial G / \partial N)_{T, P}$. Such distinction leads to the $N$-dependent difference, which one has to take into account at the simulation of two-phase $f$-states. The Gibbsian description related to the single ("test") particle moving in a homogeneous field $\mu(T, P)$ should be modified in this case by the $N$-dependent equality accordingly to the Hill's concepts [21]:

$$
(\partial \hat{\mu} / \partial N)_{T, P}=(\mu-\hat{\mu}) / N \equiv \Delta \mu / N .
$$

Such refinement of the standard test-particle methodology proposed by Widom [24], is especially important due to the mesoscopic, in fact, $(\mathrm{m}-)$ volumes of the mostly simulated VLE-diagrams. Hill [21] emphasized long ago that small system effects in the regions of phase transition and criticality are especially noticeable. In particular, an additional independent variable $N$ (discrete by nature) should be introduced in the following Clausius-Clapeyron's system of equalities. It can be considered as the formal sign of mesoscopicity in $m$-regime:

$$
\begin{gathered}
\left(\frac{\partial P}{\partial T}\right)_{N}=\frac{s_{g}-s_{l}}{v_{g}-v_{l}} \equiv \frac{\Delta s}{\Delta v} \\
\left(\frac{\partial T}{\partial N}\right)_{P}=\frac{\Delta \mu}{N \Delta s} \quad(a) \quad\left(\frac{\partial P}{\partial N}\right)_{T}=-\frac{\Delta \mu}{N \Delta v} \quad \text { (b) }
\end{gathered}
$$


where the left-hand sides and the difference $\Delta v=\left(\rho_{l}-\rho_{g}\right) / \rho_{g} \rho_{l}$ are measurable by experiment or by numerical simulation. Hence, the unmeasurable entropy-dependent differences $\Delta \mu$ and $\Delta s$ may be appropriately estimated namely by Eqs.(34-36). The implied differential equality between two uniform chemical potentials $d \hat{\mu}_{g}=d \hat{\mu}_{l}$ taken alongside of the single $P_{v}(T)$-curve was adopted in the field space to derive Eqs. $(35,36)$.

The authors of the dynamic crossover [55] have used the semiempirical interpolation function (we changed in its relaxation times the denotations of the respective densities) for the thermal energy $e\left(T, v=\rho^{-1}\right)$ :

$$
e \equiv \frac{E}{N}=k T\left[3-\left(\frac{\rho_{0}}{\rho}\right)^{3}\right] .
$$

Let us remind that the gibbsian internal energy is a function $e(v, s)$. The following contribution of compressibility at the description of a simulated potential energy by Eq.(31) has been also accepted for LJ-fluid [59]:

$$
E_{p o t}=E_{L J}(V)-E_{L J}\left(V_{0}\right)+P_{0}\left(V_{0}-V\right),
$$

where $V_{0}$ corresponds to the volume of minimal possible (negative) pressure $P_{0}$ at zero temperature. The advantages of CVL-diagram becomes also obvious in the context of such natural choice for the zero-level of potential energy. Indeed, at any given number of simulated LJ-particles $N$ with a dominance of attraction both above parameters $\left(V_{0}, P_{0}\right)$ correspond exactly to the $B$-parameters predicted in Sect. III. Three basic loci of CVL-diagram with the negative slopes coincide just at this B-point. Besides, the local definition of the calculated potential energy by Eq.(31) used in [55]is a uniform by nature. Hence, it leads sooner to the concept of Zeno-line (with its compensation of attractive and repulsive contributions) than to the presumable compensation of kinetic and potential contributions. The latter was postulated to determine the location of Frenkel's line with its positive slope in the $(\tau, \omega)$-plane.

Crossover problem in CVL-diagram. One may note that the accepted in the described procedure definition of the kinetic energy by Eq.(31) is exclusively the $T$ dependent and ig-one. It is also adopted by any simulation methodology [62-64] at the imitation of an actual fixed temperature. However, the concomitant selection [55] of the "best" critical LJ-parameters recommended in $[10,20] T_{c}^{*}=1.31, \rho_{c}^{*}=0.314$ becomes inconsistent with both GFA-estimates for Ar reported, for example, in [6]: $T_{c}^{*}=1.412 ; \quad \rho_{c}^{*}\left(A_{c}\right)=0.1905 ; \quad P_{c}^{*}\left[\rho_{g}^{*}\left(T^{*}\right) \rightarrow \rho_{c}^{*}\left(A_{c}\right)\right]=0.0785 \quad$ and $T_{c}^{*}=1.412 ;$ $\rho_{c}^{*}\left(A_{c}^{0}\right)=0.1592 ; P_{c}^{*}\left[\rho_{l}^{*}\left(T^{*}\right) \rightarrow \rho_{c}^{*}\left(A_{c}^{0}\right)\right]=0.0656$. Two variants of LJ-reduced critical density manifest the meso-fluctuations. This significant distinction and the prescribed critical "jump" of the reduced mechanical variables (Sect. II) are easily explainable. The aim of the present work is the usage of the universal realistic short- 
range FT/LJ-potential in simulations performed for any substance with the explicit CVL-diagram. Oppositely, the above "best" CP-values together with the strongly overestimated semiempirical LJ-estimates $[10,20]: P_{c}^{*}=0.126, Z_{c}=0.3063$ describe, at best, the LJ-fluid itself. One obtains the shifted values of parameters for the real Ar with its experimental CXC- [17] and CP-location: $T_{c}=150.66 \mathrm{~K} ; \rho_{c}=0.531$ $\mathrm{kg} / \mathrm{cm}^{3} ; P_{c}=4860 \mathrm{kPa} ; Z_{c}=0.2919$ [18]. GFA-estimates of the effective molecular parameters for $g$-phase of Ar: $\varepsilon / k=106.7 \mathrm{~K} ; \sigma_{g}\left(A_{c}, P_{c}\right)=0.2877 \mathrm{~nm}$ become essentially less than the conventional [23] ones: $\varepsilon / k=119.8 \mathrm{~K}$ and $\sigma=0.3405$ obtained from the theoretical second virial coefficient $B(T)$ [65]. We state now that the latters are systematically overestimated for any other fluids too.

To support this important conclusion the comparison of the short-range FT/LJpotential [6] with the different $\varepsilon, \sigma$-estimates is natural. Let us consider those following from the conventional crossover variants based on the methodology of RGtheory. The relevant $\mathrm{Il} / \mathrm{mf}$-crossover transformation is now widely discussable [2529] on the base of RG-theory of non-classical criticality. Nevertheless, the presence of $v d W$-loop at the predicted CP-temperature $T_{c}$ was noticed long ago by Wilson and Fisher. Then this mean-field feature was artificially suppressed by White and Zhang [27] at the development of global RG-expansion (GRG) for fluids. Recently the GRG-methodology with some improvements and modifications has been applied to the $n$-alkanes. The approach was used, firstly, for the rough prediction of VLEdiagram in the relatively simple hydrocarbons $\mathrm{CH}_{4}, \mathrm{C}_{2} \mathrm{H}_{6}, \mathrm{C}_{5} \mathrm{H}_{12}, \mathrm{C}_{7} \mathrm{H}_{16}$ [28]. The approach was then used to improve such VLE-predictions in $\mathrm{CH}_{4}, \mathrm{C}_{2} \mathrm{H}_{6}, \mathrm{C}_{2} \mathrm{~F}_{3} \mathrm{Cl}_{3}, \mathrm{SF}_{6}$ [29]. The results of comparison with FT/LJ-predictions are represented in Table 2.

In particular, White has demonstrated [45] the following "spectrum of possibilities" for $\mathrm{Ar}$ in comparison with the conventional but strongly overestimated values based on the second virial coefficient $B(T)$-estimates [65]: $\sigma=0.3405 \mathrm{~nm}, \varepsilon / k=119.8$ $\mathrm{K}$. One variant leads to the set of similar values $\sigma=0.3508 \mathrm{~nm}, \varepsilon / k=117.6 \mathrm{~K}$. They were obtained, however, by the artificially combined $s w / L J$-potential with the additional $h s$-diameter: $\sigma_{1}=0.3227 \mathrm{~nm}$ and the following widthes of $s w$-well: [0.3938 $\mathrm{nm}, 0.3227 \mathrm{~nm}$ ]. White predicted [45] CP- location reasonably, using then the much more wide but, simultaneously, less deep $s w$-potential for Ar: $\sigma_{1}=0.3227 \mathrm{~nm}$; $\sigma=\lambda_{s w} \sigma_{1}=0.5647 \mathrm{~nm}: \varepsilon / k=97.1 \mathrm{~K}$. The similar trends for $s w$-potential: $(0.3162$ $\mathrm{nm} ; 0.585 \mathrm{~nm} ; \varepsilon / k=69.4 \mathrm{~K})$ and $(0.3067 \mathrm{~nm} ; 0.5214 \mathrm{~nm} ; \varepsilon / k=93.3 \mathrm{~K})$ were revealed by the striking disagreement with the recommended $B(T)$-estimates [65]. We have reported in Table 2 for comparison with GFA-estimates following from Eqs. $(8,9)$ the other GRG-parameters adjusted in $[45,27-29]$ to the given set of CPparameters $\left(T_{c}, \rho_{c}, P_{c} ; Z_{c}\right)$.

One may conclude from the analysis of Table 2 (we do not comment it in detail) that GRG-methodology $[27-29,45]$ is the rather inaccurate and formidable approach to the problem of a fluid, in total, and to the problem of $f$-criticality, in particular. The very restrictive initial choice of a singular $h s$-model as well as the further usage of an 
also singular $s w$-model to accumulate the attraction contribution are not completely

Table 2. Comparison of the effective $s w$-potential parameters $(\varepsilon, \sigma, \lambda)$ used in the different GRG-variants [27-29,45] with those ( $r_{c} / \sigma$ is here unspecified) predicted for the short-range FT/LJ-potential ( $m$ - number of HC-segments in $[28,29])$.

\begin{tabular}{|c|c|c|c|c|c|c|c|c|c|}
\hline Fluid & Method & $m$ & $\sigma, \mathrm{nm}$ & $\lambda_{s w}$ & $\varepsilon / k, \mathrm{~K}$ & $Z_{c}$ & $T_{c}^{*}$ & $P_{c}^{*}$ & $\rho_{c}^{*}$ \\
\hline \multirow{3}{*}{$\mathrm{Ar}$} & GRG[45] & 1 & 0.3230 & 1.75 & 97.1 & 0.291 & 1.552 & 0.1219 & 0.2698 \\
\hline & FT/LJ & 1 & 0.2877 & $r_{c} / \sigma$ & 106.7 & 0.292 & 1.412 & 0.0785 & 0.1905 \\
\hline & LJ [20] & 1 & 0.3405 & 3.0 & 119.8 & 0.306 & 1.310 & 0.1260 & 0.3140 \\
\hline \multirow{5}{*}{$\mathrm{CH}_{4}$} & GRG[45] & 1 & 0.3550 & 1.70 & 138.0 & 0.288 & 1.381 & 0.1075 & 0.2704 \\
\hline & GRG[28] & 1 & 0.3670 & 1.44 & 168.8 & 0.293 & 1.129 & 0.1024 & 0.3093 \\
\hline & GRG[29] & 1 & 0.3590 & 1.54 & 147.9 & 0.293 & 1.131 & 0.1036 & 0.3130 \\
\hline & exp.[66] & & & & & 0.291 & $1.129^{*}$ & $0.0975^{*}$ & $0.2969 *$ \\
\hline & FT/LJ & 1 & 0.3160 & $r_{c} / \sigma$ & 135.4 & 0.290 & 1.407 & 0.0774 & 0.1901 \\
\hline \multirow{5}{*}{$\mathrm{C}_{2} \mathrm{H}_{6}$} & GRG[45] & 1 & 0.4050 & 1.63 & 264.0 & 0.285 & 1.157 & 0.0907 & 0.2750 \\
\hline & & $4 / 3$ & & 1.45 & & 0.291 & 1.263 & & 0.2382 \\
\hline & GRG[29] & $4 / 3$ & 0.3670 & 1.60 & 188.4 & 0.288 & 1.262 & 0.0877 & 0.2414 \\
\hline & exp.[66] & & & & & 0.282 & $1.263^{*}$ & $0.0793 *$ & $0.2226^{*}$ \\
\hline & FT/LJ & 1 & & $r_{c} / \sigma$ & 220.1 & 0.279 & 1.388 & 0.0754 & 0.1946 \\
\hline \multirow{5}{*}{$\mathrm{C}_{5} \mathrm{H}_{12}$} & GRG[45] & 1 & 0.5210 & 1.49 & 598.0 & 0.263 & 0.786 & 0.0563 & 0.2723 \\
\hline & GRG[28] & $7 / 3$ & 0.3931 & 1.51 & 265.0 & 0.302 & 1.773 & 0.0676 & 0.1263 \\
\hline & GRG[29] & $7 / 3$ & & & & 0.301 & 1.773 & 0.0678 & 0.1271 \\
\hline & exp.[66] & & & & & 0.269 & $1.773^{*}$ & $0.0560 *$ & $0.1176^{*}$ \\
\hline & FT/LJ & 1 & 0.4694 & $r_{c} / \sigma$ & 343.1 & 0.270 & 1.369 & 0.0735 & 0.1992 \\
\hline \multirow{4}{*}{$\mathrm{C}_{7} \mathrm{H}_{16}$} & GRG[28] & 3 & 0.3933 & 1.56 & 251.3 & 0.280 & 2.150 & 0.0549 & 0.0912 \\
\hline & GRG[28] & 3 & 0.3933 & 1.56 & 251.3 & 0.280 & 2.150 & 0.0552 & 0.0918 \\
\hline & exp.[66] & & & & & 0.282 & $2.150^{*}$ & $0.0480 *$ & 0.0793* \\
\hline & FT/LJ & 1 & 0.5229 & $r_{c} / \sigma$ & 400.0 & 0.260 & 1.350 & 0.0708 & 0.2022 \\
\hline
\end{tabular}

*) - the erroneous reduction of experimental CP-parameters taken from [66] arisen due to the inadequate choice of $(\varepsilon, \sigma)$-parameters in [29].

adequate for real fluids. They need the additional thermodynamic control of GRGtrajectory to become the reliable predictive "tool" of criticality itself and of its extension by the crossover approach.

Sengers and co-authors [25] have introduced the RG-based adjustable third $c_{t}\left(Z_{c}\right)$-coefficient in the original $\mathrm{vdW}$-methodology to compare the crossover $I l / v d W$-predictions of criticality in $\mathrm{O}_{2}, \mathrm{Ar}, \mathrm{CH}_{4}, \mathrm{C}_{2} \mathrm{H}_{6}, \mathrm{H}_{2} \mathrm{O}$. For purposes of comparison with a near-critical experiment, the reduced "shifts" between the actual \{ $\left.T_{c}, \rho_{c}, P_{c} ; Z_{c}\right\}$ - and the "imaginable" $\left\{T_{c}^{0}, \rho_{c}^{0}, P_{c}^{0} ; Z_{c}^{0}=3 / 8\right\}$-sets of CP-para-meters were expressed [25] in terms of $c_{t}\left(Z_{c}\right)$-coefficient: 


$$
\frac{T_{c}}{T_{c}^{0}} \approx 1-\frac{c_{t}}{10} \quad(a) \quad \frac{\rho_{c}}{\rho_{c}^{0}} \approx 1+\frac{c_{t}}{54} \quad \text { (b) } \frac{P_{c}}{P_{c}^{0}} \approx \frac{Z_{c}}{Z_{c}^{0}}\left(1-\frac{11 c_{t}}{135}-\frac{c_{t}^{2}}{540}\right) \quad(c) .
$$

To obtain these approximate estimates, it was supposed that:

1) far away from CP the real CXC-diameter: $\rho_{d}(T)=\left(\rho_{g}+\rho_{l}\right) / 2$ of the crossover vdW-EOS with three $\left(a, b, c_{t}\right)$-coefficients should coincide with the $m f$ diameter of vdW-EOS specified by only two $(a, b)$-coefficients;

2) the real CP-position belongs to the $m f$-vapor-pressure $P_{v}^{0}(T)$-locus in the $(P, T)$-plane (i.e. $P_{c}=P_{v}^{0}\left(T_{c}\right)$ ).

In spite of the above-mentioned absence of $m f$-CP itself in the CVL-diagram, it was the informative test for GFA-principle (see its Table 1) to compare the exact FTcorrelations $[6,8,9]$ with those following from Eq.(39):

$$
\begin{aligned}
\frac{T_{c}}{T_{c}^{0}}=\frac{27 A_{c}^{2}\left(A_{c}-2\right)}{32\left(A_{c}-1\right)^{3}} & \text { (a) } & \frac{\rho_{c}}{\rho_{c}^{0}}=\frac{3\left(A_{c}-2\right)}{2\left(A_{c}-1\right)} \\
\frac{P_{c}}{P_{c}^{0}}=\frac{27\left(A_{c}-2\right)^{2}}{4\left(A_{c}-1\right)^{3}} & \text { (a) } & \frac{Z_{c}}{Z_{c}^{0}}=\frac{16\left(A_{c}-1\right)}{3 A_{c}^{2}}
\end{aligned}
$$

The supposed by GFA-principle jump-like trend of asymptotic critical slopes: $A_{c}$ and $A_{c}^{0}=4$ corresponds to Eqs.(40,41). For the latter value $A_{c}^{0}$ one obtains the coincidence of actual CP and vdW-CP (Sect. IV). At the same time the distinction of such vdW-CP from the actually $m f$-one defined by the Andrews-van der Waals' CPconditions of Eq.(21) is crucial for CVL-diagram. Indeed, it never uses the respective mf-definition for an actual CP (implied, however, by all discussed here [25, 27-29] and by many other GRG-variants). Hence the above coincidence is a realistic asymmetric feature of the exclusively actual CP revealed by GFA-principle. Thus the dimensional CP-parameters $\left\{T_{c}, \rho_{c}, P_{c} ; Z_{c}\right\}$ are common for both asymptotic $f$-branches of CXC excluding only the values of $A_{c}$ - and $A_{c}^{0}$-slopes. Such "bifurcation" of critical slopes defines the principal distinction between a fictitious $m f$-CP from Eq.(21) and the non-classical interpretation of vdW-CP introduced by GFA-principle.

We do not comment again the reported predictive capability of RG-based crossover $\mathrm{Il} / \mathrm{mf}$-model from [25]. The reason is its obviously unrealistic (see also our recent work [6]) estimates obtained for above "shifts" by Eq.(39). It seems more interesting to emphasize that the systematic underestimation of $Z_{c}$-value by Eq.(41b) is related to its non-linear GFA-correlation with the input $A_{c}$-parameter. This observation is in contradiction to the usual PCS-assumption [11, 12] about the supposedly linear interrelation between $Z_{c}$ and Riedel's factor: $A_{c} \equiv R i$. Another interesting observation is the striking difference between an about isochoric $\rho_{c} \approx \rho_{c}^{0}$ RG-crossover trajectory and the essential "shift" (increase) of the critical density predicted by the GFA-Eq.(40b). The former effect is the obvious consequence of two abovementioned assumptions about the shape of a real fluid VLE-diagram accepted in [25]. Unfortunately, even the rather sophisticated usage of RG-theory in its alternative 
Table 3. Comparison of RG-based $\mathrm{Ib} / \mathrm{mf}$-crossover transformation [25] with that following from GFA-principle [5, 9].

\begin{tabular}{|c|c|c|c|c|c|c|}
\hline \multicolumn{5}{|c|}{ RG-crossover vdW-fluid [25] } & \multirow{2}{*}{\multicolumn{2}{|c|}{$\frac{\text { Fluid }}{\text { Experiment }}$}} \\
\hline Input & & Pre & on & & & \\
\hline$c_{t}$ & $Z_{c}^{R G}\left(c_{t}\right)$ & $\begin{array}{c}\frac{T_{c}}{T_{c}^{0}} \\
\text { Eq.(39) }\end{array}$ & $\begin{array}{c}\frac{\rho_{c}}{\rho_{c}^{0}} \\
\text { Eq.(39) }\end{array}$ & $\begin{array}{c}\frac{P_{c}}{P_{c}^{0}} \\
\text { Eq.(39) }\end{array}$ & $\begin{array}{c}\text { Substan } \\
\text { ce }\end{array}$ & $\begin{array}{l}Z_{c} \\
{[12]}\end{array}$ \\
\hline 0.0 & 0.375 & 1 & 1 & 1 & $\mathrm{Ar}$ & 0.291 \\
\hline 0.2 & 0.349 & 0.977 & 1.004 & 0.900 & $\mathrm{O}_{2}$ & 0.292 \\
\hline 0.5 & 0.309 & 0.943 & 1.009 & 0.786 & $\mathrm{CH}_{4}$ & 0.290 \\
\hline 1.0 & 0.255 & 0.892 & 1.019 & 0.624 & $\mathrm{C}_{2} \mathrm{H}_{6}$ & 0.279 \\
\hline 1.5 & 0.217 & 0.853 & 1.028 & 0.513 & $\mathrm{H}_{2} \mathrm{O}$ & 0.229 \\
\hline
\end{tabular}

\begin{tabular}{|c|c|c|c|c|c|c|c|}
\hline \multicolumn{8}{|c|}{ GFA-crossover of real fluids $[5,9]$} \\
\hline \multicolumn{3}{|c|}{ Input } & \multicolumn{5}{|c|}{ Prediction } \\
\hline $\begin{array}{c}\text { Substan } \\
\text { ce. }\end{array}$ & $\begin{array}{c}Z_{c} \\
{[12]}\end{array}$ & $\begin{array}{c}A_{c} \\
{[12]}\end{array}$ & $\begin{array}{l}Z_{c}\left(A_{c}\right) \\
\text { Eq. }(41 b)\end{array}$ & $\begin{array}{c}\delta Z_{c} \\
\%\end{array}$ & $\begin{array}{l}\frac{T_{c}}{T_{c}^{0}} \\
\text { Eq.( } \\
40 \mathrm{a})\end{array}$ & $\begin{array}{r}\frac{\rho_{c}}{\rho_{c}^{0}} \\
\text { Eq. }(40 b)\end{array}$ & $\begin{array}{c}\quad \frac{P_{c}}{P_{c}^{0}} \\
\text { Eq. }(41 \mathrm{a})\end{array}$ \\
\hline $\mathrm{Ar}$ & 0.291 & 5.76 & 0.287 & -1.05 & 0.976 & 1.185 & 0.885 \\
\hline $\mathrm{O}_{2}$ & 0.292 & 5.92 & 0.281 & -3.85 & 0.973 & 1.195 & 0.871 \\
\hline $\mathrm{CH}_{4}$ & 0.290 & 5.90 & 0.282 & -2.92 & 0.974 & 1.194 & $0 / 873$ \\
\hline $\mathrm{C}_{2} \mathrm{H}_{6}$ & 0.279 & 6.40 & 0.264 & -5.50 & 0.966 & 1.222 & 0.830 \\
\hline $\mathrm{H}_{2} \mathrm{O}$ & 0.229 & 7.86 & 0.222 & -3.02 & 0.946 & 1.281 & 0.718 \\
\hline
\end{tabular}

GRG-variants shares the first questionable assumption leading to the artificial global "symmetrization" of both CXC-branches. In other words, one obtains (see Figs. represented in $[28,29])$ the certain "graphic superposition" of the classical binodal with its $m f$-CP top and exponent $\beta_{0}=1 / 2$ (predicted by the known unified SAFT-EOS, for example), from one hand, and the non-classical CXC-variant with its respective fluctuation flattening due to RG-exponent $\beta \simeq 0.326$ from another. Such "superposition" is considered, from our viewpoint, without the correct account for the GFA-nature of real fluids. The CVL-diagram eliminates this restriction of RG-theory by the simultaneous account for both effects (see also [26]). The notions of $m f$-CP and classical binodal/spinodal construction never appear in the GFA-methodology. The close location of above lines in the $(P, T)$-plane well-established by many authors, poses the fundamental supercritical fluids SCF-problem. Could one recognize in this region the common underlying molecular-level peculiarities of $f$-states in the thermodynamic second derivatives and transport coefficients, which lead to their recognizable by experiment or simulation extrema? If the answer is here positive, one can divide the SCF-region onto the subregions of SCF-strata with the certain persistent type of a molecular-based structure, at least, in $m$-regime of criticality. 
At the construction of CVL-diagram in g-regime (Fig. 2) we have supposed that the rejection from a unified EOS and from its unavoidably $m f$-CP $\left(T_{c}^{0}, P_{c}^{0}, \rho_{c}^{0} ; Z_{c}^{0}\right)$ defined by Eqs. $(21 \mathrm{a}, \mathrm{b})$ is the necessary step. The FT-model let us to separate by $q s p-$ locus and by the concomitant $m l$-locus the global $f$-strata at any temperature. They were termed by use of capital letters GAS, FLUID, STABLE AND METASTABLE LIQUID to emphasize the distinction from the traditional location of $g, f, l$-states in the restricted region of VLE-diagram. The latter is completely based on the binodal/spinodal's $m f$-concept rejected by FT-EOS (1). GFA-principle admits [5, 9] the $f$-dependent discontinuity in its well-definable [37-43] $T$-dependent FTcoefficients determined alongside the entire $q s p$-locus.

The underlying discontinuity in the effective molecular diameters of collisions $\sigma_{f}$ following from Eqs.(3) and $(9 \mathrm{a}, \mathrm{b})$ makes the other common for both $f$-phase effective parameter of well-depth $\varepsilon_{g, l}$ in Eq.(8) to be of importance at the universal molecular interpretation of the thermodynamic fluctuations in $m$-regime. It seems naturally to suppose $[5,42,43]$ that the only third effective parameter of well-width $r_{c}$ should be also necessary common for f-phase and enough to interpret any mesoscopic thermodynamic phenomena. One of the main aims of such finite-range three-parameter FT/LJ-potential $\phi_{F T / L J}^{f}\left(r ; \varepsilon, \sigma_{f}, r_{c}\right)$ is namely the simulation in SCFstates. The goal is the more precise determination of the qsp-location shown in Fig. 2.

These notes actuate the introduction of a normalized PCS-fluctuation variable of the type that introduced by Eq.(11) in LSB-EOS (10) [1]:

$$
Z_{c} \cdot \Delta \equiv Z_{c} \frac{\left\langle(\Delta N)^{2}\right\rangle}{\bar{N}}=\tau\left(\frac{\partial \omega}{\partial \pi}\right)_{\tau>1}=\frac{\Delta \cdot\left(T_{c}^{*}-1\right)}{T_{c}^{*}} .
$$

The last equality based on Eq.(8) is essential to recognize its generalized form. It is accepted [43] to specify the reference infinite-range LJ-potential $\phi_{L J}\left(r ; \sigma_{f}, \varepsilon, r_{c} \rightarrow \infty\right)$ at the unit reduced temperature $T^{*}=\tau \cdot T_{c}^{*}=1$. It was shown earlier by FT-model that the average LJ-virial $[w]$ determined per a translation degree of freedom leads to the physically-meaningful description of any $f$-states in $g$-regime:

$$
Z_{L J}-1=\frac{[W]}{3 N k T}=\frac{[w]}{k T} \text {. }
$$

This FT-correlation established between the negative average derivative of a potential energy (i.e. force field of the aforementioned attraction) multiplied by the average local interparticle distance, from one hand, and the average kinetic energy, from another, can be related to a single CP-states itself. To our mind, the given coupled FT-correlation of $[w] / k T$-terms and its thermodynamic manifestation by the fluctuation coefficient $c_{f}(T)$ from Eq.(2) is somewhat more fundamental notion in comparison with the conventional analysis of LJ-model in terms of potential and kinetic energies. The latter is, of course, restricted by the adopted ig-model of a simulated temperature $\left(k\langle T\rangle=m_{0}\left\langle u^{2}\right\rangle / 3\right)\left(k\langle T\rangle=m_{0}\left\langle u^{2}\right\rangle / 3\right)$ performed in accordance 
with Eq.(32) at the pseudo-ig-constraints of Zeno-line $\left(Z_{L J}=1,[w]=0\right)$ due to the standard equipartition theorem [21-23]. We have returned to the original vdW-EOS (4) reduced now by its mf-CP-parameters to illustrate the other interesting consequences of GFA-principle by Fig. 4. The obvious advantage of the rejection from the $m f$-constraint introduced by the Andrews-van der Waals' definition in Eq.(21) is a direct possibility to compare the fluctuation contours of vdW-fluid and real fluid Ar:

$$
\tau_{\Delta}=\frac{6 Z_{c} \cdot \Delta(3-\omega)^{2}}{24 Z_{c} \cdot \Delta-(3-\omega)^{2}} .
$$

For this aim we have calculated and represented by continuous line the same fixed $\Delta$-values $\Delta=2.5$ discussed earlier by Nishikawa and co-authors [56] at the $m f$ constraint $Z_{c}^{0}=3 / 8$. The added dashed lines correspond to the choice $Z_{c}=0.2919$ for Ar. The qsp-locus termed the fluctuation ridge by above authors remains invariable in all projections of Fig. 4.

Conclusion. The predictive superiority of FT-model has been demonstrated by this review in the step-by step manner. The detailed explanation of the mesoscopic fluctuation concepts is represented. The numerical results corroborate the approach of FT-model globally without the restrictions of non-classical criticality or the specially mean-field low-temperature behavior predicted by any unified EOS-type.

\section{References:}

1. Lienhard J.H., Shamsundar N.and Biney P.O. Spinodal lines and equations of state: a review // Nucl.Eng. and Design. -1986. - Vol. 95. P. 297-314.

2. Shamsundar N. and Lienhard J.H. Equations of state and spinodal lines - a review // Nucl.Eng. and Design. - 1983. - Vol. 141. - P. 269-287.

3. Corty D.S. and Debenedetty P.G. A computational study of metastability in vaporliquid equilibrium // Chem.Eng.Sci. - 1994. - Vol. 49. - P. 2717-2734.

4. Kido A., Kitao O. and Nakanishi K. Can the "van der Waals loop" vanish? // Chem.Phys.Lett. - 1992. - Vol. 199. - P. 403-407.

5. Rogankov V.B. Fluctuational-thermodynamic interpretation of small angle X-ray scattering experiments in supercritical fluids // Fluid Phase Equilibria. - 2014. - Vol. 383. - P. 115-125.

6. Rogankov O.V., Rogankov V.B. Can the Boyle and critical parameters be unambiguously correlated for polar and associating fluids, liquid metals, ionic liquids? // Fluid Phase Equilibria. - 2017. - Vol. 434. - P. 200-210.

7. Rogankov V.B., Fedyanin V.K. Fluctuational theory of media with essential spatialtemporal inhomogeneity // Theor. and Math. Phys. - 1993. - Vol. 97, №1 . - P. 1-21.

8. Rogankov V.B., Boshkov L.Z. Gibbs solution of the van der Waals-Maxwell problem and universality of the liquid-gas coexistence curve // Phys.Chem.Chem.Phys. 2002. - Vol. 4. - P. 873-878.

9. Rogankov V.B., Levchenko V.I. Global asymmetry of fluids and local singularity in the diameter of the coexistence curve // Phys.Rev.E - 2013. - Vol. 87. - P.052141. 

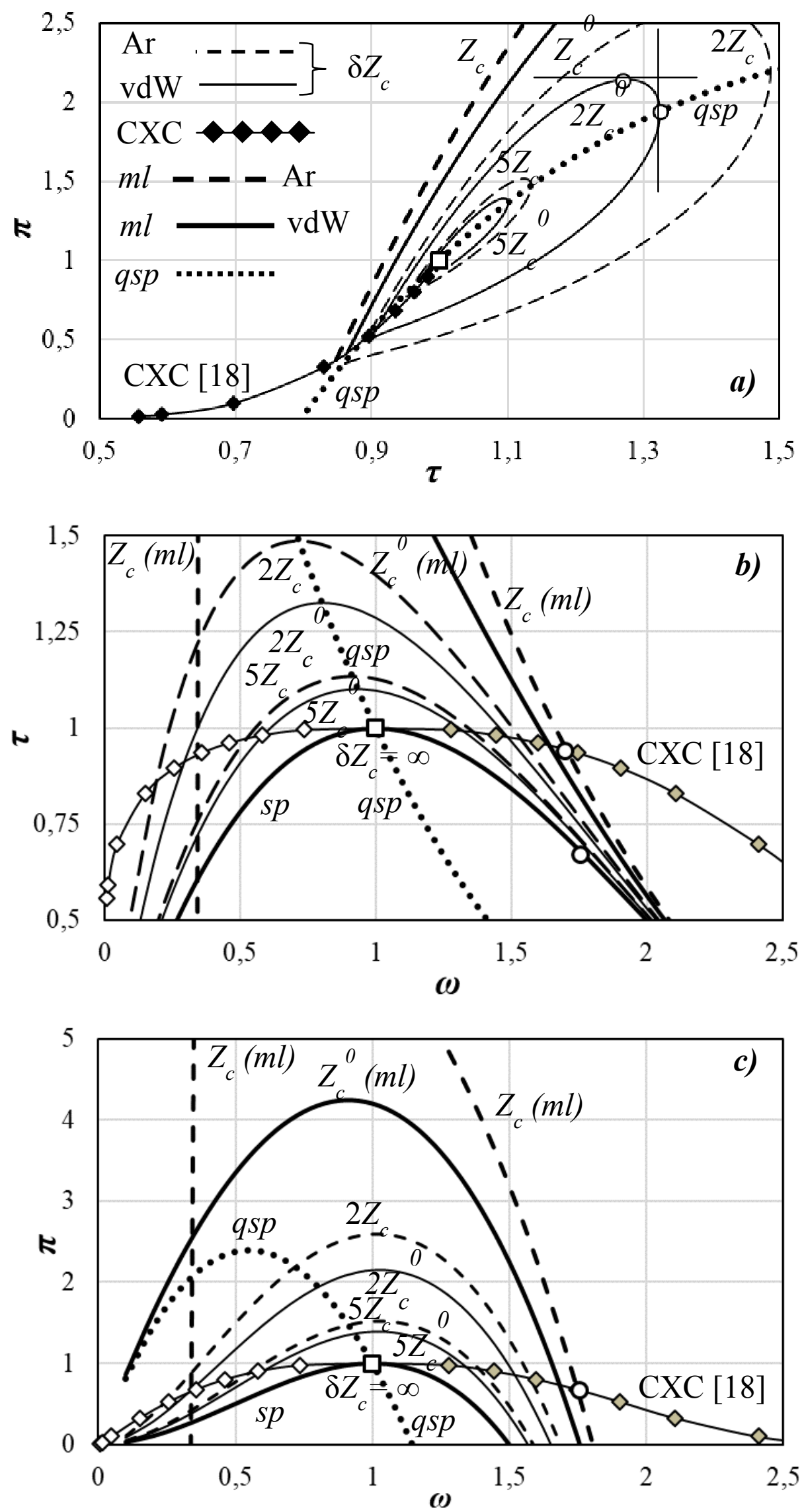

Fig. 4. The forced elimination of the crossover range (see Fig. 2) between the actual $\mathrm{CP}$ and the fictitious $m f-\mathrm{CP}$ by their artificial matching for Ar and vdW-fluids leads, as a rule, to the popular speculations [46-59] about the supercri-tical third$\operatorname{order}(g, l)$-transition. 
10. Johnson J.K., Zollweg J.A., Gubbins K.E. The Lennard-Jones equation of state revisited // Mol.Phys. - 1993. - Vol. 78 (3). - P. 591-618.

11. Filippov L.P. Methods of Calculation and Prediction for Thermophysical Properties, - Moscow Univ. Publ., 1988.

12. Sterbaček Z., Biskup B., Tausk P. Calculation of Properties using CorrespondingStates Methods. - Elsevier Science Publishing Company, Amsterdam, Oxford, NewYork, 1979.

13. D.Ben-Amotz and D.R.Herschbach Correlation of Zeno $(Z=1)$ line for supercritical fluids with vapor-liquid rectilinear diameters // Israel J. of Chemistry (online). 1996. - Vol.30(1-2).

14. Apfelbaum E.M., Vorob'ev V.S., Martynov G.A. Universal triangle of states for liquid and vapor // Russ.J.Struct.Chem. - 2006. - Vol.47. - P. 113-121.

15. Kulinskii V.L. The critical compressibility factor value. Associative fluids and liquid alkali metals // J.Chem.Phys. - 2014. - Vol. 141. - P. 054503.

16. Estrada-Torres R., Iglesias-Silva G.A., Ramos-Estrada M., Hall K.R. Boyle temperatures for pure substances // Fluid Phase Equil. - 2007. - Vol. 258. -P. 148-154.

17. Gilgen R., Kleinrahm R., Wagner W. Measurment and correlation of the (pressure, density, temperature) relation of argon: I The homogeneous gas and liquid regions in the thermodynamic range from $90 \mathrm{~K}$ to $340 \mathrm{~K}$ at pressures up to $12 \mathrm{MPa} / /$ J.Chem.Thermodyn. - 1994. - Vol. 26. - P. 383-398; II Saturated liquid and saturated-vapor densities and vapor pressures along the entire coexistence curve, ibid. 1994. - Vol. 26. - P. 399-413.

18. Stewart R.B., Jacobsen R.T. Thermodynamic properties of saturated argon // J.Phys.Chem.Ref.Data. - 1989. - Vol. 18. - P. 679-683.

19. Umirzakov I.H. The method to define critical volume of one-component substance using Boyle temperature and critical pressure // Butlerov Communications. - 2015. Vol. 44. - P. 118-121..

20. Lotfi A., Vrabec J., Fischer J. Vapour liquid equilibria of the Lennard-Jones fluid from NPT plus test particle method // Mol.Phys. - 1992. - Vol. 76. - P. 1319-1333.

21. Hill T.L. Thermodynamics of Small Systems. - Dover Publ. Inc., New York, 1994.

22. Stanley H.E. Introduction to Phase Transition and Critical Phenomena. - Clarendon Press, Oxford, 1971.

23. Balescu, R. Equilibrium and Nonequilibrium Statistical Mechanics. - Eds. J.Willey\&Sons, New York-London-Sydney-Toronto, 1975.

24. Widom B. Some topics in theory of fluids // J.Chem.Phys. - 1963. - Vol. 39. - P. 2808-2813.

25. Wyczalkowska A.K., Sengers J.V., Anisimov M.A. Critical fluctuations and the equation of state of van der Waals // Physica A334. - 2004. - P.482.

26. Wang J., Anisimov M.A. Nature of vapor-liquid asymmetry in fluid criticality // Phys.Rev. - 2007. - Vol. E75. - P. 051107.

27. White J.A., Zhang S. Renormalization group theory for fluids // J.Chem.Phys. 1993. - Vol. 99. - P. 2012-2019.

28. Zhao W., Wu L., Wang L., Li L., Cai J. Critical asymmetry in renormalization group theory for fluids // J.Chem.Phys. - 2013. - Vol. 138. - P. 234502. 
29. Wang L., Zhao W., Wu L., Li L., Cai J. Improved renormalization group theory for critical asymmetry of fluids // J.Chem.Phys. - 2013. - Vol. 139. - P. 124103.

30. Fisher M.E., Zinn S.-Y. The shape of the van der Waals loop and universal critical amplitude ratios // J.Phys. A Math.Gen. - 1998. - Vol. 31. - P. L629-L635.

31. Fedyanin V.K., Rogankov V.B. Scaling equation of the thermodynamical surface of a fluid. I Close vicinity of the equilibrium state // Phys.Lett. A160- 1991. - Vol.3.

32. Rowlinson J.S. Liquids and Liquid Mixtures. - London, Batherworths Sci. Publ., 1959. $-360 \mathrm{p}$.

33. Rowlinson J.S., Widom B. Molecular Theory of Capillarity. - London, Oxford University Press, 1982.

34. Rowlinson J.S. van der Waals revisited // Chem.Brit. - 1980. - Vol. 16. - P. 32-35.

35. Penrose O., Lebiwitz J.L. Towards a rigorous molecular theory of metastability// Fluctuation Phenomena (edited by E.Montroll and J.Lebowitz). Chapt. 5, NorthHolland, Amsterdam, 1987.

36. Skripov V.P. Metastable Liquids. - Wiley, New York, 1974.

37. Rogankov V.B. Asymmetry of heterophase fluctuations in nucleation theory // $\mathrm{Nu}-$ cleation Theory and Applications (edited by J.W.P.Schmelzer, G.Röpke and V.B.Priezjev) Chapt. 22, Dubna, JINR, 2011.

38. Mazur V.A., Rogankov V.B. A novel concept of symmetry in the model of fluctuational thermodynamics // J.Mol.Liq. - 2003. - Vol. 105/2-3. - P. 165-177.

39. Rogankov V.B., Byutner O.G., Bedrova T.A., Vasiltsova T.V. Local phase diagram of binary mixtures in the near-critical region of solvent // J.Mol.Liq. - 2006. - Vol. 127. - P. 53-59.

40. Rogankov V.B. Equation of state for ionic liquids // High Temperatures. - 2009. Vol. 47. - P. 656-663.

41. Rogankov V.B., Levchenko V.I., Kornienko Y.K. Fluctuational equation of state and hypothetical phase diagram of superheated water and two imidazolium-based ionic liquids // J.Mol.Liq. - 2009. - Vol. 148, №1 . - P. 18-23.

42. Rogankov V.B., Levchenko V.I. Towards the equation of state for neutral $\left(\mathrm{C}_{2} \mathrm{H}_{4}\right)$, polar $\left(\mathrm{H}_{2} \mathrm{O}\right)$, and ionic $\left([\mathrm{bmim}]\left[\mathrm{Bf}_{4}\right],[\mathrm{bmim}]\left[\mathrm{Pf}_{6}\right],[\mathrm{pmmim}]\left[\mathrm{Tf}_{2} \mathrm{~N}\right]\right)$ liquids // Journal of Thermodynamics. - Volume 2014, Article ID 496835, 15 pages.

43. Rogankov V.B. Scaling model of low-temperature transport properties for molecular and ionic liquids // Journal of Termodynamics, Volume 2015, Article ID 208486, 11 pages.

44. Huang Y.-H., O'Connell J.P. Corresponding states correlation for the volumetric properties of compressed liquids and liquid mixtures // Fluid Phase Equil. -1987. Vol. 37. - P. 75-84.

45. White J.A. Volumetric properties of fluids calculated by Renormalization Group theory // Proceedings of the Twelfth Symposium on Thermophysical Properties, Boulder, Colorado, USA, 1994.

46. Semenchenko V.K. Selected chapters of theoretical physics. - Education Publ., M., 1966.

47. Woodcock L.V. Observation of a thermodynamic liquid-gas critical coexistence line and supercritical fluid phase bounds from percolation transition loci // Fluid Phase Equilibria. - 2013. - Vol. 351. - P. 25-33. 
48. Woodcock L.V. Gibbs density surface of water and steam: $2^{\text {nd }}$ debate on the absence of van der Waals" "Critical point" // Natural Science. - 2014. - Vol. 6. - P. 411-432.

49. Griffiths R.B., Wheeler J.C. Critical points in multicomponent systems // Phys.Rev. A2. - 1970. - P. 1047-1063.

50. W.F. van Gunsteren, H.C.Berendsen Computer simulation of molecular dynamics: methodology, applications, and perspectives in chemistry // Angew.Chem.Int.Ed.Engl. - 1990. - Vol. 29. - P. 992-1023.

51. Bernal J.D. The structure of liquids // Proceedings of the Royal Society (London). 1962. - A280. - P. 299-322.

52. Widom B.J. Phase Transitions and Critical Phenomena. - Eds. C.Domb and M.S.Green, Phase Transitions and Critical Phenomena, v.2, Academ. Press, Waltham.

53. Simeoni G.G., Bryk T., Gorelli F.A., Krisch M., Ruocco G., Santoro M., Scopigno T. The Widom line as the crossover between liquid-like and gas-like behavior.

54. Brazhkin V.V, Fomin Y.D., Lyapin A.G., Ryzhov V.N., Tsiok E.N. Widom line for the liquid-gas transition in Lennard-Jones system // J. Phys. Chem. - 2011. - B115. - P. 14112-14115.

55. Brazhkin V.V., Fomin Y.D., Lyapin A.G., Ryzhov V.N., Trachenko K. Two liquid states of matter: a dynamic line on a phase diagram // Physical Review E85. - 2012. - P. 031203.

56. Nishikawa K, Morita T. Fluid behavior at supercritical states studied by small-angle X-ray scattering // J. of Supercritical Fluids. - 1998. - Vol. 13. - P. 143-148.

57. Arai A.A., Morita T., Nishikawa K. Investigation of structural fluctuation of supercritical benzene by small-angle X-ray scattering // J. Chem. Phys. - 2003. - Vol. 119. - P. 1502-1509.

58. Nishikawa K., Kusano K., Arai A.A. Density fluctuation of a van der Waals fluid in supercritical state // J. Chem. Phys. - 2003. - Vol. 118. P. 1341-1346.

59. Ma T., Wang $S$. Third-order gas-liquid phase transition and the nature of Andrews critical point // AIP Advances. - 2011. - Vol. 1. - P. 042101.

60. Hoover W.G., Stell G., Goldmark E., Degani G.D. Generalized van der Waals equation of state // J.Chem.Phys. - 1975. - Vol. 63. - P. 5434-5438.

61. Hagen M.H.J., Frenkel D. Determination of phase diagram for the hard-core attractive Yukawa system // J.Chem.Phys. - 1994. - Vol. 101. - P. 4093-4097.

62. W.F. van Gunsteren, H.C.Berendsen Computer simulation of molecular dynamics: methodology, applications, and perspectives in chemistry // Angew.Chem.Int.Ed.Engl. - 1990. - Vol. 29. -P. 992-1023.

63. Panagiotopoulos A.Z. Molecular Simulations of Phase Equilibria // Supercritical Fluids. / Eds. E.Kiran, J.M.H. Levelt-Sengers, Kluwer Academic Publishers, Netherlands, 411-437, 1994.

64. B.Smit, Ph. de Smedt and D.Frenkel Computer simulations in the Gibbs ensemble // Mol.Phys. - 1989. - Vol. 68. - P. 931-950.

65. Hirschfelder J.O., Curtiss C.F., Bird B.B. Molecular Theory of Gases and Liquids. J.Wiley and Sons, N.Y., 1954.

66. Vargaftik N.B. Handbook of physical properties of liquids and gases. - Hemisphere, Washington, 1983. 
67. Pitzer K.S. Some Interesting Properties of Vapor-Liquid or Liquid-Liquid Coexistence Curves for Ionic and Non-Ionic Fluids // Thermochimica Acta. - 1989. - Vol. 139. - P. 25-32.

68. Rogankov O.V. Shvets M.V., Kalinchak V.V., Sergeeva A.E., Rogankov V.B., Levchenko V.I. Elongate coexistence curve and its curvilinear diameter as factors of global fluid asymmetry // Physics of Aerodisperse Systems. - 2017. - № 54, C. 8-29.

69. Rogankov V.B., Shvets M.V., Rogankov O.V., Chikunkova T.A. Supercritical heterogeneous nanostructure of fluids 1 . Diagram of fluctuation transitions in non-gibbsian phases // Physics of Aerodisperse Systems. - 2019. - №56. - C. 14-29.

70. Rogankov V.B., Shvets M.V., Rogankov O.V., Chikunkova T.A. Supercritical heterogeneous nanostructure of fluids 2. Its potential impact on creation of coupled stirlings with intermediate regeneration of heat // Physics of Aerodisperse Systems. 2019. - №56. - C. 30-48.

\title{
Роганков В.Б., Швець М.В., Роганков О.В. Мезоскопічна метастабільна рідина в конгруентній пара-рідинній діаграмі аргону (огляд ФТ-моделі)
}

\begin{abstract}
АНОТАЦІЯ
Традииійне об'єднання понять класичної метастбільності і некласичної скейлінгової критичної області в рамках єдиного рівняння стану з середньо-польовою, $m$. зв. Ендрю - ван-дер-Ваальсівською, критичною точкою повинне бути піддане додатковому аналізу. Його метою є встановлення реалістичної розмарованої структури мезоскопічної рідкої фази. Вона, імовірно, існує в широкому інтервалі температур, локалізованих між майже нульовою $і$ бойлівською точками Р,Т-діаграми. Запропоновано альтернативне прийня-тому обтрунтування наявності безперервних кросоверних границь діаграми. Вони розділяють окремі структурні підобласті. Район гетерогенної рідкої фази, який покриває глобально весь такий інтервал, доречно назвати не-гіббсівською фазою. Це пояснюється ї̈ дискретною гетерогенно-інтерфазною структурою і не вимагає посилань на явище спінодального розпаду. Відповідна область метастабільного розшарування утворена трьома підобластями: 1) надкритичною; 2) підкритичною і 3) сублімаційної конденсованої фази для формально нестисливої рідини. Остання визначена одночасною фіксацією двох екстенсивних масштабів об'єму $і$ числа частинок. Ї̈ розтамування на конгруентній пара-рідинній діаграмі обмежене введеною новою границею флуктуаційної метастабільності, а також відомою Зено-лінією. Таким чином, всі підобласті включають область м'якого флюїду з переважанням міжчастинкового притягання. Решта конгруентної діаграми відповідає газовій $і$ твердій підобластям (кристалічної або аморфної фаз) в твердому флюїді по класифікаиії, запропонованій Бен Амочем і Хершбахом.
\end{abstract}

Ключові слова: мезоскопічна рідина, гетерогенна інтерфаза, границі метастабільності. 